\title{
Fast restoration for out-of-focus blurred images of QR code with edge prior information via image sensing.
}

\author{
CHEN, R., ZHENG, Z., YU, Y., ZHAO, H., REN, J. and TAN, H.-Z.
}

(C) 2021 IEEE. Personal use of this material is permitted. Permission from IEEE must be obtained for all other uses, in any current or future media, including reprinting/republishing this material for advertising or promotional purposes, creating new collective works, for resale or redistribution to servers or lists, or reuse of any copyrighted component of this work in other works. 


\title{
Fast Restoration for Out-of-focus Blurred Images of QR Code with Edge Prior Information via Image Sensing
}

\author{
Rongjun Chen ${ }^{1,2}$, Zhijun Zheng ${ }^{1}$, Yongxing $\mathrm{Yu}^{1}$, Huimin Zhao ${ }^{1}$, Jinchang Ren ${ }^{1,3}$, Senior \\ Member, IEEE, and Hong-Zhou Tan², Senior Member, IEEE
}

\begin{abstract}
Out-of-focus blurring of the QR code is very common in mobile Internet systems, which often causes failure of authentication as a result of a misreading of the information hence adversely affects the operation of the system. To tackle this difficulty, this work firstly introduced an edge prior information, which is the average distance between the center point and the edge of the clear QR code images in the same batch. It is motivated by the theoretical analysis and the practical observation of the theory of CMOS image

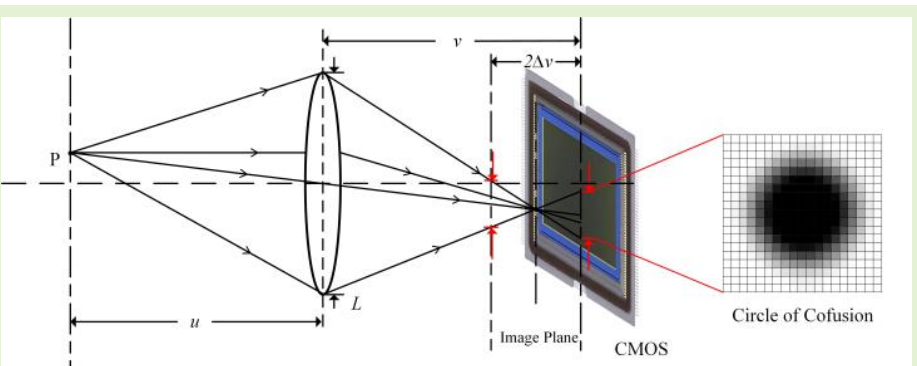
sensing, optics information, blur invariants, and the invariance of the center of the diffuse light spots. After obtaining the edge prior information, combining the iterative image and the center point of the binary image, the proposed method can accurately estimate the parameter of the out-of-focus blur kernel. Furthermore, we obtain the sharp image by Wiener filter, a non-blind image deblurring algorithm. By this, it avoids excessive redundant calculations. Experimental results validate that the proposed method has great practical utility in terms of deblurring quality, robustness, and computational efficiency, which is suitable for barcode application systems, e.g., warehouse, logistics, and automated production.
\end{abstract}

Index Terms-Image Sensing; Optical Imaging; Out-of-focus Blurring; QR Code Images; Edge Prior Information

\section{Introduction}

$\mathrm{W}$ ith the rapid development of the Internet of Things technology and the information industry, Quick Response (QR) code, which is initially designed to track inventory in vehicle parts manufacturing, has been widely used in various fields due to their large information capacity, wide coding range, high reliability and low cost [1-4]. However, in practical application scenarios such as industrial production lines, once the external factors occur (such as loosening, vibration of lens or inaccurate focus), it is prone to generate out-of-focus blurred images of $\mathrm{QR}$ code. This

This work is supported by the National Natural Science Foundation of China under Grant 62072122, the Innovation Team Project of the Education Department of Guangdong Province under Grant 2017KCXTD021, the Key Lab Project of the Education Department of Guangdong Province under Grant 2019KSYS009, the Project for Distinctive Innovation of Ordinary Universities of Guangdong Province under Grant 2018KTSCX120, the Ph.D. Start-up Fund of Natural Science Foundation of Guangdong Province under Grant 2016A030310335, the Science and Technology Planning Project of Guangdong Province under Grant 2017B090908006, and the Guangdong Colleges and Universities Young Innovative Talents Projects under Grant 2018KQNCX138. (Corresponding authors: Jinchang Ren; Hong-Zhou Tan)

1. School of Computer Science, Guangdong Polytechnic Normal University, Guangzhou 510665, China

2. School of Electronics and Information Technology, Sun Yat-sen University, Guangzhou, 510006, China (e-mail: issthz@mail.sysu.edu. $\mathrm{cn}$ )

3. National Subsea Centre, Robert Gordon University, Aberdeen AB107AQ, U.K. (email: jinchang.ren@ieee.org) widespread degradation usually leads to difficulties in object recognition and scene interpretation. It adversely affects the decoding and the extraction of $\mathrm{QR}$ codes and leads to inaccurate representation of the information. In this regard, in view of the high-efficiency requirements of real-time systems, fast restoration plays a prominent role in many industrial applications such as merchandise flow systems, commodity production systems and package sorting systems [5].

When the blurring is uniform and spatially invariant, we can use the convolution operation to model the blurring process. In general, the model of image degradation is:

$$
g(x, y)=h(x, y) * f(x, y)+n(x, y)
$$

where $f(x, y)$ is the original image, $h(x, y)$ is the point spread function (PSF), * is the convolution operator, $n(x, y)$ is the image noise, and $g(x, y)$ is the blurred image after degradation. It has been shown theoretically and empirically that this general property of blurring process is applicable to many images [6].

The out-of-focus blur occurs in the imaging process when the image plane is away from the ideal sensors reference plane. The PSF in Fourier optics theory can demonstrate the relationship between the object and the image, which is frequently used in out-of-focus image deblurring. If the image noise is not considered, the PSF represents a diffuse spot pattern formed by a photon passing through the optical 
imaging system in the process of imaging. In other words, the PSF can be regarded as a finite impulse response filter for each point light source. Its convolution operation will cause the image quality to deteriorate, i.e., how blurred images generate. And image blurring often shows that many more sensitive details to human vision are lost, such as edges and corners, i.e., the resolution of the image is reduced. The PSF of the defocus degradation model used in our paper is defined as:

$$
h(x, y)=\left\{\begin{array}{cc}
\frac{1}{\pi R^{2}}, & x^{2}+y^{2} \leq R^{2} \\
0, & \text { otherwise }
\end{array}\right.
$$

where $R$ is the radius of the circle of confusion $[7,8]$.

Image restoration is one of the hot and intractable topics in image processing where many methods have been proposed in the last decades. Van et al. [9] introduced a regularization method for the deblurring of QR code images simultaneously. However, if a prior information could be introduced to incorporate with nonlinear constraints, it would have better results. Based on the black and white constraint characteristics of barcode images, Liu et al. [10] proposed a minimal filter based on incremental constraints to restore defocused twodimensional codes, which used the binary characteristics of the barcode. However, since the finder pattern varies from different types of 2D barcode, their method will be limited in use. Based on the linear singularity characteristics of the barcode images, Tiwari et al. [11] introduced the Ridgelet Transform (RT) and RBF neural network for barcode deblurring. There were some complex transformation operations in the algorithm, which made it unable to operate in limited computing power. Michaeli et al. [12] introduced the internal region reconstruction technology into the field of image deblurring, but its fuzzy kernel estimation had certain volatility, which affected the quality of image restoration. Pan et al. [6] proposed a deblurring algorithm with low sparsity prior based on dark channels. By introducing a minimum operator to solve the non-convex linear optimization problem caused by the sparsity of dark channels, the natural image could be deblurred. However, the noise would seriously affect the estimation of the derived fuzzy kernel. On the basis of the
Pan's algorithm, Liu et al. [4] introduced the binary characteristics of QR codes and constructed a new constraint item to achieve double-layer constraints on QR images, and had an excellent performance against ordinary blur kernels and multiple noises effects. This algorithm was more effective than previous ones, but it still failed to improve the speed of blind deblurring. Nah et al. [13] achieved deblurring of dynamic scenes by simulating multi-scale loss functions, simultaneously based on multi-scale convolutional neural networks. However, a large number of training samples and training time were the disadvantages of this algorithm.

At present, image deblurring algorithms mainly consider the processing of natural images or text pictures. When it is applied to QR code image deblurring, it does not fully consider its binary and step edge characteristics, ignoring the error correction mechanism of the barcode image itself and resulting in poor recovery effect or slow speed. To tackle this difficulty, we proposed a fast restoration algorithm based on edge prior information. With the advancement of image sensing technology and step edge features, the out-of-focus blurred QR code images can be restored fleetly. The strength is that it can be suitably applied to warehouse auto-sorting systems.

The workflow diagram of a typical system is shown in Fig. 1. In this system, parcels with QR codes are transported to the sorting system through the conveying device. To start with, the package is recognized by the infrared sensor, and its main function is to activate the shooting function of the area scan camera. In addition, this system mainly has a diversion link, i.e., whether the internal information of the QR code can be correctly recognized. If the answer is 'yes', the system will send the package to the sorting part for shipment and delivery. Otherwise, it will be diverted to the exception-handling part, where costly manual intervention is needed. By introducing more effective deblurring and other related steps for improved QR code reading, cases that need to be passed to the exception-handling part can be minimized hence the overall save of the costs and improvement of the efficiency and customer experience. Furthermore, the sorting equipment obtains the predetermined results according to the cargo information and classifies the goods accordingly. Since the auto-sorting system classifies goods according to the relevant QR codes, quickly and accurately classifying the QR codes is

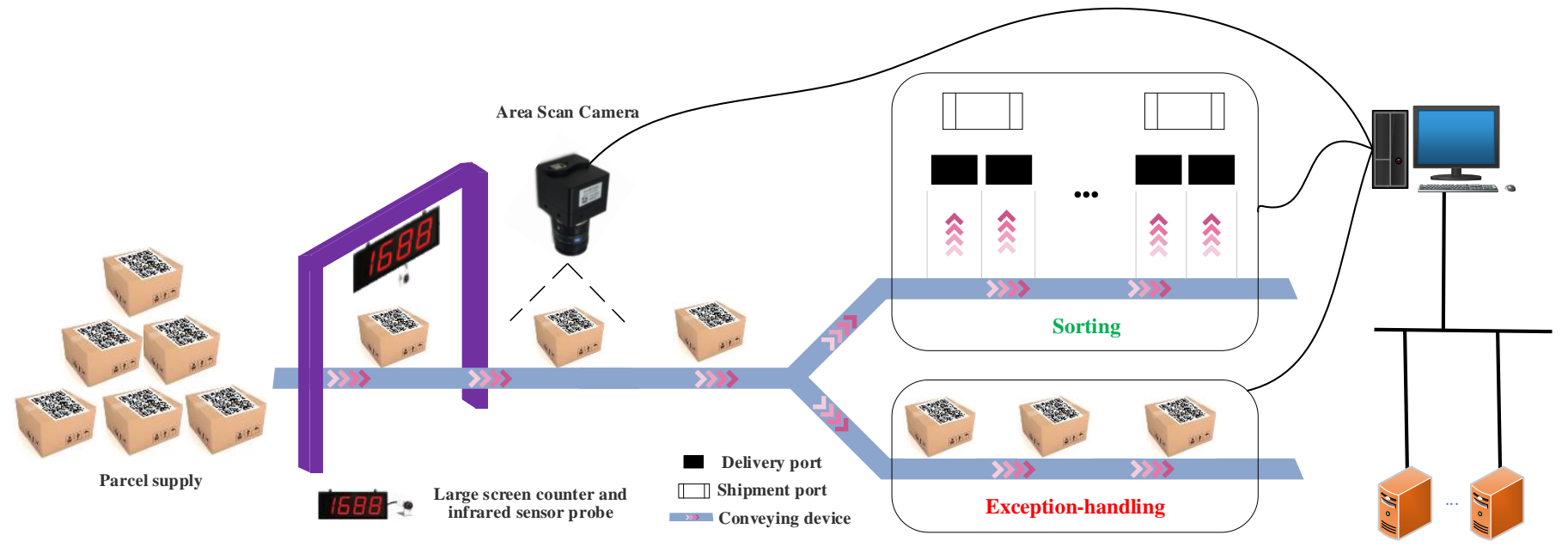

Fig. 1. Diagram of warehouse automatic sorting system. 
the key to such systems. How to accurately and rapidly identify the QR code depends on the efficacy and efficiency of the system, as well as the capacity and scale of the warehouse [14]. However, as captured moments are ephemeral and difficult to reproduce, the out-of-focus blur caused by lens loose or lens vibration in the optical imaging process has introduced a lot of unnecessary time cost to the warehouse management system. Therefore, we introduce edge prior information. The accuracy of this information ensures the robustness of the restoration effect. Moreover, we fully consider the step edge characteristics of the QR code to achieve a rapid restoration. Theoretical analysis and experimental observation prove that the proposed method can effectively solve problems such as long operation time and low robustness, and further improve the industry's actual expectation.

The rest of the paper is organized as follows: we introduce related work in Section II. In Section III, we introduce the technical details of the proposed method. In Section IV, we compared it with other different blind deblurring algorithms and analyzed the experimental results. Finally, Section $V$ concludes this paper.

\section{RELATED WORK}

Recently, with the support of various prior images and blur kernels, image deblurring technology has made great progress. According to whether the blur kernel is known in advance or not, these methods can be divided into two categories [15], i.e., non-blind image deblurring and blind image deblurring. Detailed discussions are given as follows.

\section{A. Non-blind Image Deblurring}

For non-blind image deblurring, the blur kernel has been given, and the issue it confronts is to use the blur kernel to recover a latent sharp image from the blurred observation. Intuitively, it is a precarious process and is easily disturbed by noise. Even a small amount of noise will cause serious distortion in the estimation, a phenomenon called the illposedness. To solve this ill-posed problem, conventional methods are often applied including the Wiener filtering [16], the Richardson-Lucy [17,18], constrained least squares filtering [19] et al. Moreover, image deblurring can also be modeled as an optimization problem, such as end-to-end interpretable learning [20], hybrid non-convex second-order total variation [21], fully convolutional networks [22], et al.

\section{B. Blind Image Deblurring}

Blind image deblurring refers to the restoration of latent images from blurred observations without knowing the information of the blur kernel. By estimating or trying the known blur kernel, the blind image deblurring can be converted to a non-blind image deblurring problem. A unified framework [23] and generalized Gaussian blur kernels [7] benefit the image deblurring process. In other regard, many existing methods have introduced complex image priors in blind image restoration as a joint optimization problem by penalizing image blurriness and improving the image sharpness. Some natural image priors have been used to reduce the ill-posedness, such as $L_{0}$-regularized intensity and gradient prior [24], normalized color-line priors [25], dark channel prior [6], enhanced low-rank prior [26], graph-based prior [27], multi-scale latent structure prior [15], local maximum gradient prior [28] and deep priors [29], etc. These priors act as a regularizer in the optimization model and guide the solver to converge to a potentially clear image. However, these methods can effectively reduce the ill-posed of blind image restoration algorithms, but the priors are usually nonconvex, leading to complex optimization algorithms. Moreover, they are usually computationally expensive.

In addition, the parametric assumptions about the blur kernel can greatly improve the robustness of blind image deblurring [7, 30], which is the use of partially known or inaccurate degradation models in the largest posterior framework that helps to solve image restoration principal algorithm [31]. For other deep learning and other methods, they use methods to contain a large number of real images sets and training sets, such as deep multi-scale convolutional neural network [13], spatially variant recurrent neural networks [32], conditional adversarial networks [33] and scale-recurrent network [34], etc. The characteristics of the training data are usually set to affect the entire model greatly. A common drawback of deep learning algorithms is that their capability always depends on the consistency between the training data and the test data although these data-driven methods can produce a good performance in various situations.

\section{QR code Image Deblurring}

At present, many studies have proposed different algorithms for the deblurring of $\mathrm{QR}$ code images. An alternating minimization restoration model based on binary characteristic and $L_{0}$ norm minimization [4] and a method based entirely on Kullback-Leibler divergence [35] for blind deblurring and denoising have been proposed for considering the binary and symbolic characteristics of QR code images. On the other hand, a regularization approach [9] and an anisotropic total variation regularized $L^{1}$-approximation method [36] have been proposed to address the ill-posed problem of image restoration and prevent image overfitting. In addition, doubly convolutional neural network [5] had been proposed to leverage the deep learning technique to bridge the gap between traditional model-based methods and the requirement of reversing the blurry 2D barcode images. However, algorithms that use deep learning methods to deal with the blurring of QR code images are relatively rare, because the performance of deep learning models for deblurring QR code images in industrial automation still needs to be explored.

The premise of these algorithms is that the PSF is unknown. Therefore, in the guidance of these algorithms, we give priority to obtaining the parameters of the disc defocus PSF to calculate the PSF. This measure transforms the blind restoration problem into the non-blind restoration problem, so as to realize the rapid restoration of QR code images. The next section will introduce the proposed method.

\section{The Proposed Method}

Before obtaining PSF, we need to determine the step or the approximate step of the edge contained in the image. The position of the edge can be obtained by detecting the edge of the defocused $\mathrm{QR}$ code by a detection operator based on the 
image step edge characteristics. Moreover, combining the edge prior information, the maximum second derivative of the column position of the edge is obtained. Finally, $R$ can be obtained by further calculation. This section depicts the optical theory of out-of-focus blurred images and analyzes the step edge and the edge prior of the $\mathrm{QR}$ codes, and then further proposes the recovery process of this paper.

\section{A. Optical Theory of Out-of-focus Blurred Images of QR Code}

Sensor is an important component of information systems, and the technology is mainly used for information collection. At present, the sensor is developing from the traditional discrete type to the direction of monolithic integration, intelligence, networking, and systemization. And the image sensor is one of the main components in digital imaging systems (such as digital cameras or camcorders) [37]. The photoelectric effect occurs when an external light shines on a pixel array, generating a corresponding charge within the pixel cell. The image signal is transmitted to the corresponding analog signal processing unit and analog to digital converter through the signal bus of the respective column and then converted into digital image signal output. The sensor has the advantages of random window reading ability, optimized exposure control, system complexity, and reliability [38]. In view of its relatively superior performance, CMOS image sensor is widely used in various fields.

Based on theoretical analysis and practical observation, outof-focus occurs in an optical imaging system when the distance between the image plane and the sensors plane is large enough. Lebl et al. [39] mentioned that any PSF with central symmetry has blur invariants under convolution. Since the defocus blur of the camera is similar to the circle of confusion, there is good invariance performance when the object is in the common plane. The PSF of out-of-focus blur has the following properties:

$$
\begin{aligned}
& h(x, y)=h(-x,-y) \\
& \int_{-\infty}^{\infty} \int_{-\infty}^{\infty} h(x, y) d x d y=1
\end{aligned}
$$

Flusser et al. $[40,41]$ original defined the blur invariants.

Assuming that the image $f(x, y)$ is a piecewise function, and it is not all zeros in area $D$ of the $x-y$ plane, there is the following $B\left(D_{p}, D_{q}\right)$ :

$$
B\left(D_{p}, D_{q}\right)=\left\{\begin{array}{cc}
0, & \left(D_{p}+D_{q}\right) \% 2=0 \\
\mu_{D_{p} D_{q}}-\frac{1}{\mu_{00}} \sum_{n=0}^{p} \sum_{\substack{m=0 \\
0 n n+m p_{p}+D_{q}}}^{q}\left(\begin{array}{c}
D_{p} \\
n
\end{array}\right)\left(\begin{array}{c}
D_{q} \\
m
\end{array}\right) B\left(D_{p}-n, D_{q}-m\right) \sqcap \mu_{n m}, & \text { Otherwise }
\end{array}\right.
$$

where $\mu_{D_{p} D_{q}}$ is the central moment. For $h(x, y)$ and any $D_{p}$ and $D_{q}$, there is $B\left(D_{p}, D_{q}\right)^{(f)}=B\left(D_{p}, D_{q}\right)^{\left(f^{*} h\right)}$. Other blur invariants constructed by formula (5) also have the invariance of translation, rotation, scaling, and tilt. Moreover, Chen [42] proposed a practical method based on the model of optical out-of-focus imaging and blurred image processing of quickly and automatically measuring the focal length. this method can estimate the distance between diffuse light spots with high accuracy so as to realize the measurement of automatic focus more accurately and quickly. It effectively proves the invariance of the center of the diffuse light spots.

In the imaging process of the lens, ensuring the maximum consistency between the image and the object is the purpose of studying the imaging system. In this part, the relevant knowledge of optical information theory is used to guide the study of optical imaging on the basis of Shannon's theorems. Random process and probability statistics are the core content of information theory. How to connect the concept of probability with imaging problems is an important basis for explaining optical imaging problems on the basis of information theory. Assuming that the light intensity distribution on the object surface is $O S\left(x_{i}\right)$, using the law of large numbers and normalizing, the relationship between the light intensity distribution on the object surface and the probability of photons can be expressed as:

$$
p\left(x_{i}\right)=O S\left(x_{i}\right)
$$

This formula shows that the light intensity of the object plane at $x_{i}$ is equal to the probability $p\left(x_{i}\right)$ of photons appearing in the neighborhood of $x_{i}$. In the same way, the light intensity on the image plane satisfies:

$$
p\left(x_{i}\right)=I\left(y_{i}\right)
$$

Among them, $I\left(y_{i}\right)$ is the light intensity distribution of the
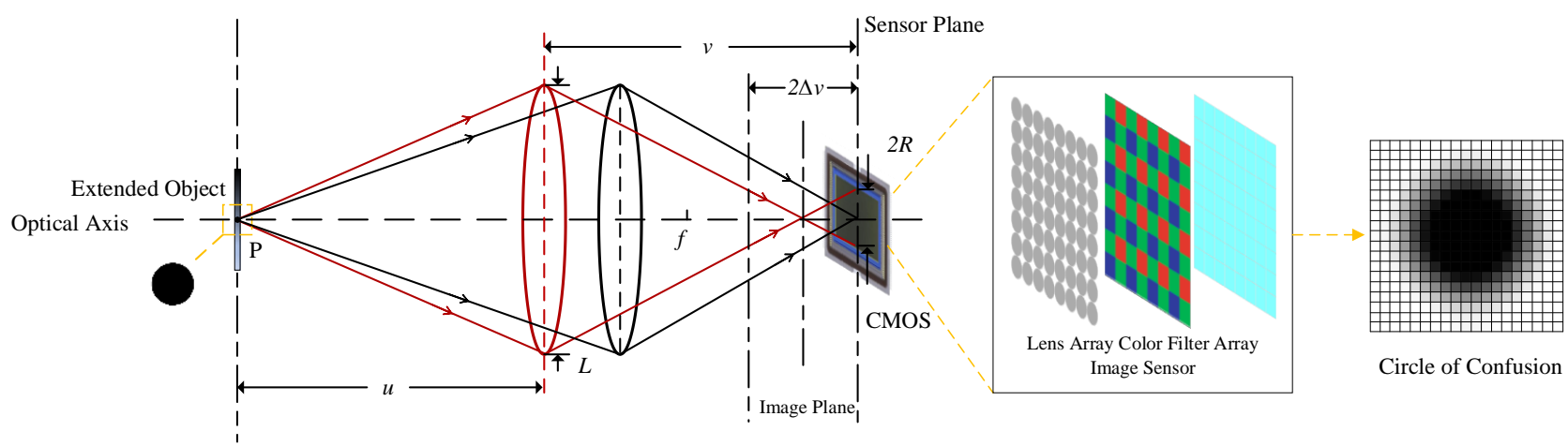

Circle of Confusion

Fig. 2. Lens optics imaging model related to out-of-focus blur. 
image plane. According to formulas (6) and (7), assuming that $p\left(y_{i} / x_{i}\right)$ represents the point spread function of the object point $x_{i}$ at a certain image point $y_{i}$, then:

$$
p\left(y_{i} / x_{i}\right)=h\left(y_{i}, x_{i}\right)
$$

By introducing total probability theorem in probability theory, the photon generated and emitted in the neighborhood of $x_{i}$ is defined as event $\mathrm{NH}$, and the received photon in the neighborhood of $y_{i}$ on the image plane is defined as event $P$, then:

$$
\begin{gathered}
P=P \cap N H_{1}+P \cap N H_{2}+\cdots+P \cap N H_{N} \\
p(P)=\sum_{n=1}^{N} p\left(P / N H_{n}\right) p\left(P_{n}\right)
\end{gathered}
$$

$N$ is the sum of all facets in the object plane. Combining formulas (6-8), formula (10) can be expressed as the imaging equation of the optical system in the discrete case:

$$
I\left(y_{m}\right)=\sum_{n=1}^{N} h\left(y_{m}, x_{n}\right) O S\left(x_{n}\right)
$$

Assuming that $h\left(y_{m}, x_{n}\right)$ has space translation invariance, we transform formula (11) into an imaging equation under continuous conditions, and express it in integral form:

$$
I(y)=\int h(y, x) O S(x) d x
$$

This formula is the equation of the imaging system.

Under the guidance of the theory of blur invariants, the invariance of the center of the diffuse light spots and the information theory of lens imaging, Fig.2 illustrates a simplified lens optics imaging model related to out-of-focus blur. In the field of view, spot $P$ is imaged on the sensor plane through Lens $L$. When the lens is loose or vibrates, i.e., when the image distance becomes larger, a circle of confusion with a radius of $R$ will be formed on the reference plane, an image sensor with a lens array color filter array. The size of the circle of confusion is related to the position of the focus point - the more blurred the image, the larger the circle of confusion $[7,8]$.

From the perspective of geometric optics, the ideal thin lens satisfies the Gaussian imaging constraint [43]:

$$
\frac{1}{f}=\frac{1}{u}+\frac{1}{v}
$$

where $u$ is the object distance, $v$ is the image distance, and $f$ is the focal length of the optical system. As seen from the geometric relationship in Fig. 2, the radius of the circle of confusion has the following relationship with the defocus amount:

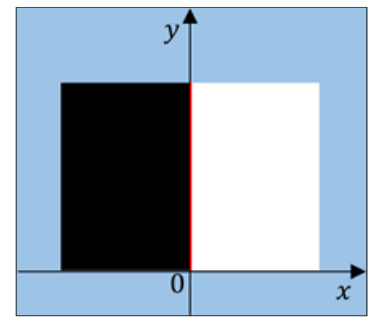

(a)

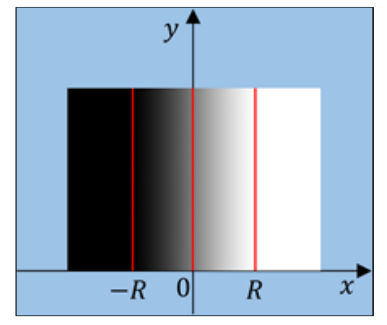

(b)
Fig. 3. Step edge under different conditions. (a) step edge of a clear image (b) step edge of a blurred image.

$$
\frac{2 R}{L}=\frac{\Delta v}{v}
$$

Combining Eqs. (13) and (14), we can get:

$$
R=\frac{L(u-f) \Delta v}{2 f u}
$$

where $R$ is the radius of the circle of confusion, $L$ is the diameter of the lens, and $\Delta v$ is the distance between the sensor reference plane and the image plane, i.e., the defocus of the optical system.

\section{B. Step Edge of QR Code}

In general, a clear QR code is a binary image consisting of black-and-white pixel blocks. Such a binary image is rich in step edge information, but it should be noted that step edge information changes with the process of defocusing. But at present, the algorithms often pay too much time because they involve iterative blurred kernel estimation $[6,44,45]$, require a large number of training samples and complex training processes $[13,31-34,46]$. As seen, these algorithms ignore the features and the error correction mechanism of QR codes.

The step edge, an obvious feature of QR codes, refers to the area where the gray value of the image undergoes a step change, which represents the information characteristics of the image. In general images, the step edge information is weakened due to the uniform distribution of gray values, which brings certain difficulties to the acquisition of edge information. However, the QR codes naturally contain rich step-edge information because they are composed of black and white pixel blocks, as shown in Fig. 3.

Fig. 3 shows the grayscale changes in the edge area of the QR code before and after defocusing. Fig. 3(a) is the step edge of a clear image while Fig. 3(b) is the step edge of a blurred one. In addition, Fig. 3(a) is the adjacent black and white modules in the original clear image. The dividing line between them can be regarded as an ideal step edge. The corresponding mathematical expression is:

$$
f(x, y)=a+b u(x)
$$

where $u(x)=\left\{\begin{array}{cc}1, & x \geq 0 \\ 0, & \text { Otherwise }\end{array}\right.$, it is the standard Heaviside

function along the $\mathrm{X}$-axis. $a$ and $b$ are integers. And $a$ is the image gray value in the negative direction of the $\mathrm{X}$-axis, while 


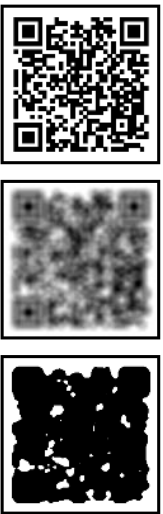

(a)

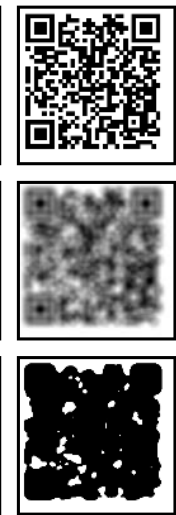

(b)
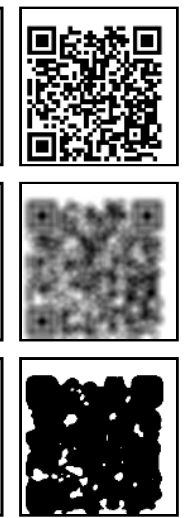

(c)

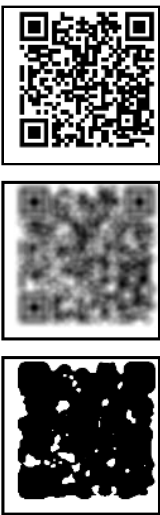

(d)
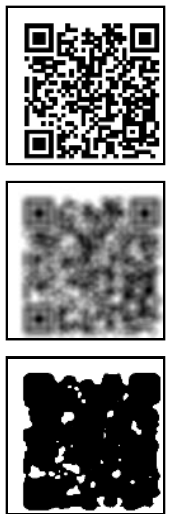

(e)
Fig. 4. Three types of the same $Q R$ code. The upper layer is clear $Q R$ code, the middle layer is blurred QR code, and the bottom layer is binary QR code. (a) in the center (b)upper left (c)lower left (d)upper right (e)lower right.

$b$ is the jump value of the image after the step change, and their value range are $0 \leq a \leq 255,0 \leq b \leq 255$, and $0 \leq a+b \leq 255$. As shown in Fig. 3(b), it is the defocused image of the clear black and white module in Fig. 3(a). When a clear QR code becomes defocused, the ideal step edge is transformed into a blurred edge area, which is the blurred step edge. This is the unique structural feature of the QR code, and the boundary width of the blurred area in the blur step edge can be calculated through this feature to obtain $R$.

\section{Edge Prior Information}

In different situations, the $\mathrm{QR}$ codes obtained in the common batch are often not in the center of the image, and they will translation in different directions, such as upper left, lower left, upper right, and lower right, as shown in Fig. 4. The upper layer shows the clear QR code images after translation in different directions. The middle layer displays the out-of-focus blurred images of the QR code corresponding to the upper layer, and their blur radius are 11 identically. The bottom layer uses a function to obtain a threshold value, and the images of the middle layer are converted to binary images. At this moment, we customize the vertices of the QR code,

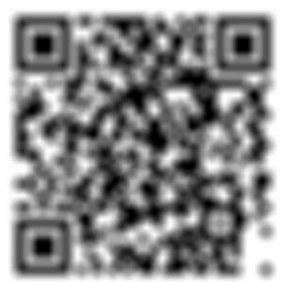

(a)

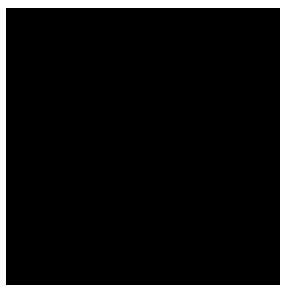

(b)

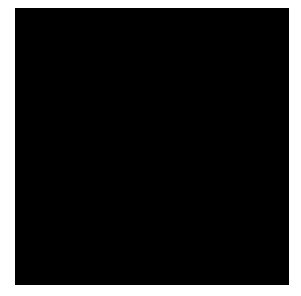

(c)

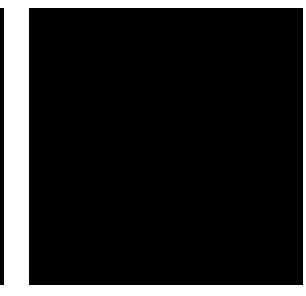

(d)
TABLE ।

PARAMETER ANALYSIS OF QR CODE TRANSLATION IN DIFFERENT DIRECTIONS

\begin{tabular}{lllllll}
\hline \hline & & Center & $\begin{array}{c}\text { Upper } \\
\text { left }\end{array}$ & $\begin{array}{c}\text { Lower } \\
\text { left }\end{array}$ & $\begin{array}{c}\text { Upper } \\
\text { right }\end{array}$ & $\begin{array}{c}\text { Lower } \\
\text { right }\end{array}$ \\
\hline & clear & 20 & 12 & 10 & 30 & 31 \\
$x_{1}$ & blurred & 15 & 7 & 5 & 25 & 26 \\
& binary & 17 & 9 & 7 & 27 & 28 \\
& clear & 282 & 274 & 272 & 292 & 293 \\
$x_{2}$ & blurred & 286 & 278 & 276 & 296 & 296 \\
& binary & 285 & 277 & 275 & 295 & 296 \\
& clear & 282 & 274 & 272 & 292 & 293 \\
$y_{3}$ & blurred & 286 & 278 & 276 & 296 & 296 \\
& binary & 285 & 277 & 275 & 295 & 296 \\
Center & clear & 151.0 & 143.0 & 141.0 & 161.0 & 162.0 \\
point(x-co- & blurred & 150.5 & 142.5 & 140.5 & 160.5 & 161.0 \\
ordinate) & binary & 151.0 & 143 & 141.0 & 161.0 & 162.0 \\
Prior & clear & 131.0 & 131.0 & 131.0 & 131.0 & 131.0 \\
distance & clarn & & & & &
\end{tabular}

namely the upper left vertex $\left(x_{1}, y_{1}\right)$, the upper right vertex $\left(x_{2}, y_{2}\right)$, and the lower left vertex $\left(x_{3}, y_{3}\right)$. The relevant parameters obtained through experiments are shown in Table I, where the value of prior distance is equal to the value of center point (x-coordinate) minus the value of $x_{1}$.

It can be concluded from the analysis of experimental data in Table I, compared with natural images, in the common batch of QR code images, the distance between the center point (x-coordinate) and $y_{1}$ is equal, which is the prior information of this work. It is of great significance for accurately calculating the blur radius. It can be known from Section III .B that the black and white binarization characteristics of $\mathrm{QR}$ codes make the edges easier to obtain. However, the more blurred the image, the more difficult it is. Therefore, according to the grayscale characteristics of $\mathrm{QR}$ codes, this paper uses an operator based on grayscale images for edge detection (Fig. 5), finds the optimal one, and then analyzes edge information with different blurred degrees (Fig. 6). Among them, the edge detection operator mainly includes

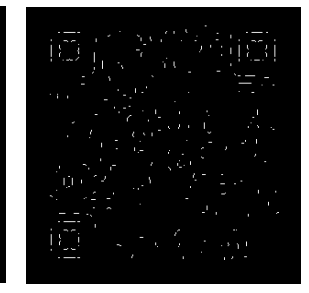

(e)

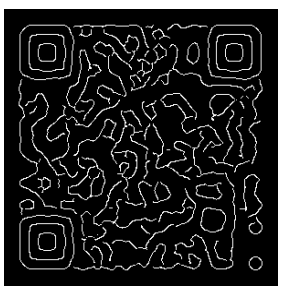

(f)
Fig. 5. Edge detection of blurred QR code. (a) blurred image (b) Sobel (c) Roberts (d) Prewitt (e) LoG (f) Canny.

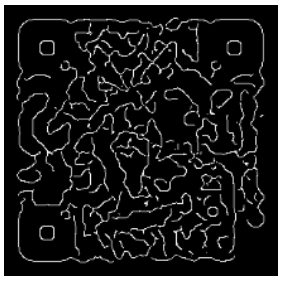

(a)

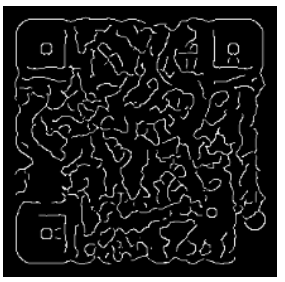

(b)

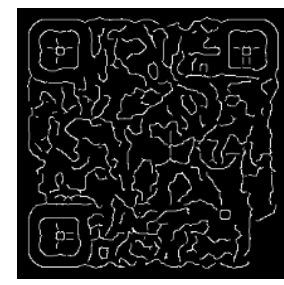

(c)

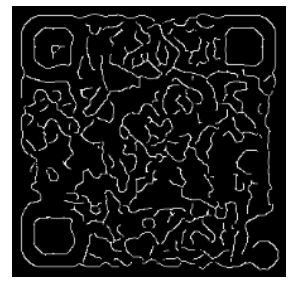

(d)

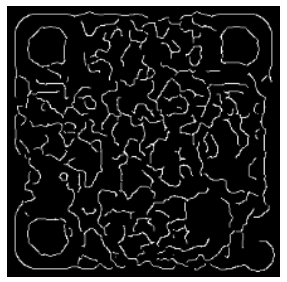

(e)

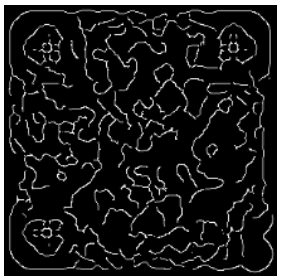

(f)
Fig. 6. Canny operator detects blurred images of different blur radius. (a) $R=10$ (b) $R=12$ (c) $R=14$ (d) $R=16$ (e) $R=18$ (f) $R=20$. 


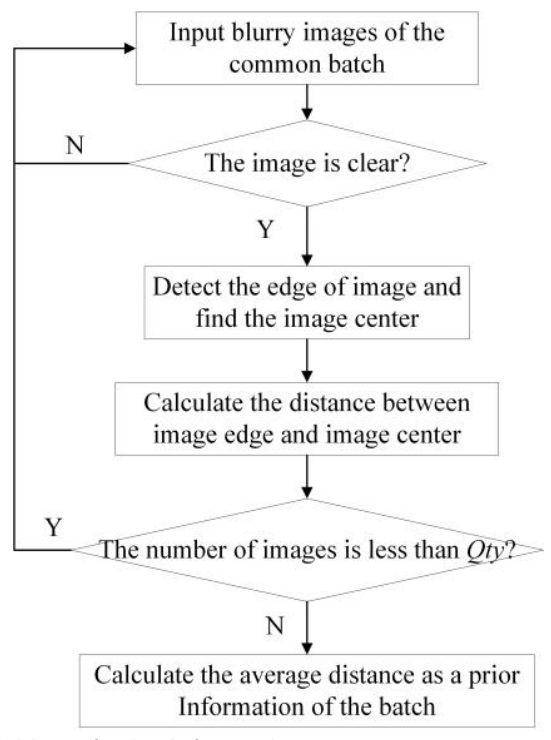

Fig. 7. Acquisition of prior information.

first-order and second-order. The traditional first-order detection differential operators include Prewitt [47], Roberts [48], Sobel [49], etc. These operators usually have corresponding calculation templates. The differential image operators for second-order detection include LoG [48] and Canny [50].

It can be seen from Fig. 5 that when the QR code is blurry, only the Canny detection operator can more completely detect the edge of the QR code image. Therefore, in the proposed method, the Canny operator is used to detect and obtain edge prior information and edge. However, as the ambiguity deepens, when the ambiguity is high, as shown in Fig. 6, the position of the edge is spreading out. Therefore, based on the edge features of $\mathrm{QR}$ codes, this paper adds edge prior information. The main steps are shown in Fig. 7.

Under the guidance of the optical theory of out-of-focus blurred images of QR code in Section III.A, we can extend that under the common batch of images, its geometric centrality remains unchanged. Therefore, in Fig. 7, prior is obtained from clear QR code images of the common batch. Among them, we use Zxing 3.4.0 to evaluate the clarity of the input image. When the image reaches a certain definition, the distance between the image edge and the center point is calculated. Last but not least, the number of clear images Qty is set to obtain a more accurate level mean value, which is the distance prior of the batch.

\section{Proposed Deblurring Algorithm Using Prior Information}

To overcome the shortcomings of the traditional estimation algorithms, such as poor anti-noise performance, inaccurate estimation of blur radius, and low algorithm stability, we proposes a fast restoration method for severely out-of-focus blurred images based on edge prior information by using the step edge characteristics and gradient features. The restoration process of the proposed algorithm is shown in Fig. 8. It mainly includes the following steps.

Step 1: Preprocessing of the input image. Firstly, we select the image that needs to be deblurred and determine whether

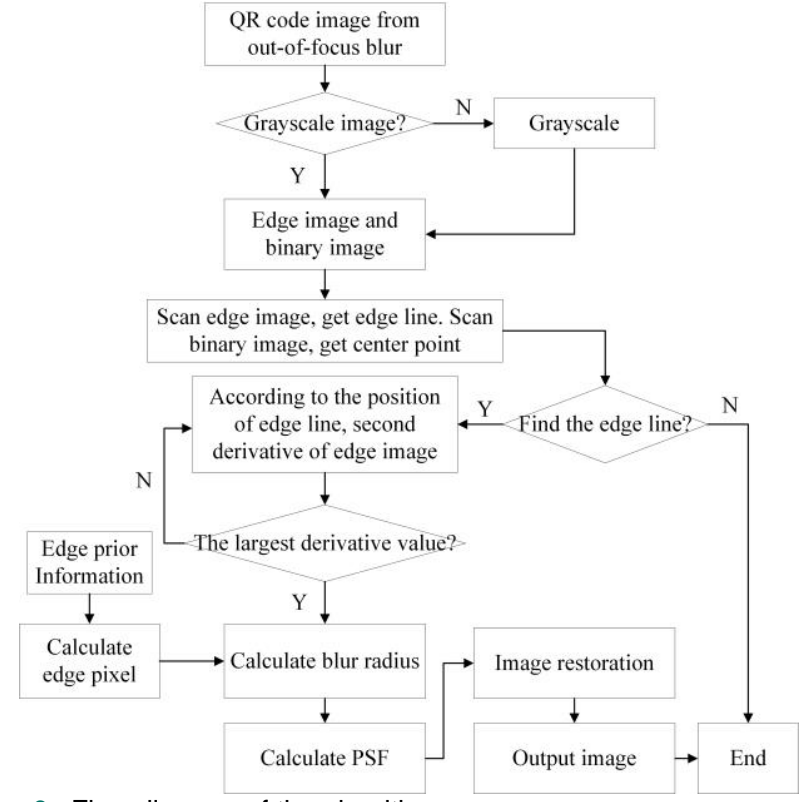

Fig. 9. Flow diagram of the algorithm.

the image is a grayscale image. If not, we will perform the grayscale processing on the image to obtain image $F$.

Step 2: Use the Canny operator to detect image $F$, then scan from top to bottom and left to right. Then we use the position of the left line as the edge to obtain the iterative image $A$. The purpose of this step does not need to calculate the value of the entire image $F$ and then accurately obtain the value of the defocus radius, which reduce the amount of calculation and increase the speed of the algorithm effectively. At the same time, we binary $F$ using Otsu algorithm to obtain the $O\left(x_{0}, y_{0}\right)$ accurately where $x_{0}=x_{2}-x_{1}$ and $y_{0}=y_{3}-y_{1}$, which is experimented by Table I in Section III.C.

Step 3: Calculate the two-step gradient of iterative image $A$. Assuming that a function has a continuous first partial derivative in the plane area, the gradient at any point can be expressed by formula (17):

$$
\nabla f(x, y)=\frac{\partial f}{\partial x} i+\frac{\partial f}{\partial y} j
$$

Combining the prior information of the $\mathrm{QR}$ code edge generated in Section III.C, we assume that the distance prior obtained is $d$. Therefore, the edge of the image when it is clear is $x_{c}=x_{0}-d$. Moreover, we look for the $Q$ with the largest rate of change of the derivative value along the normal direction of the column where the starting position is located. Combining the image gradient theory described above and the formula (1), (2), and (16), we get the formula is as follows:

$$
\begin{aligned}
& \frac{\partial g(x, y)}{\partial x}=h(x, y) * \frac{\partial f(x, y)}{\partial x}=h(x, y) * b \delta(x) \\
& =\int_{-\infty}^{\infty} \int_{-\infty}^{\infty} h(\alpha, \beta) b \delta(x-a) d \alpha d \beta \\
& =b \int_{-\infty}^{\infty}\left[\int_{-\infty}^{\infty} h(\alpha, \beta) b \delta(x-a) d \alpha\right] d \beta=b \int_{-\infty}^{\infty} h(x, \beta) d \beta
\end{aligned}
$$




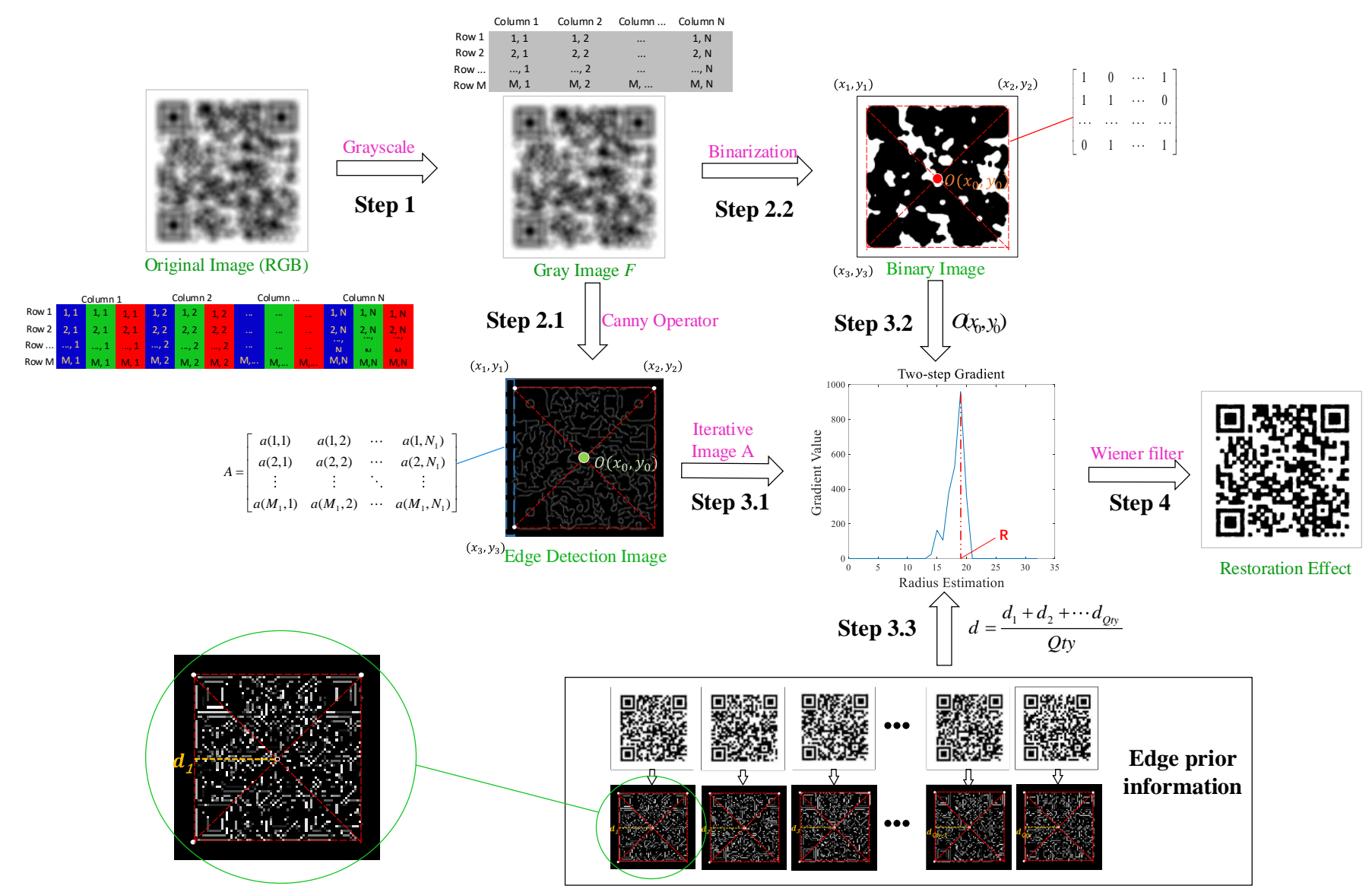

Fig. 8. Restoration process of the proposed algorithm.

where $\delta(x)$ is the unit impulse response function along the $\mathrm{X}$ axis. According to the point with the maximum derivative change rate is the maximum value point of the two-step gradient of the blurred image, we continue to calculate the derivative of $x$ by formula (18):

$$
\frac{\partial^{2} g(x, y)}{\partial x^{2}}=\frac{b \partial\left[\int_{-\infty}^{\infty} h(x, \beta) d \beta\right]}{\partial x}=\frac{b \partial\left[\int_{-\infty}^{\infty} h(x, y) d y\right]}{\partial x}
$$

Combining formula (2), we get:

$$
\frac{\partial^{2} g(x, y)}{\partial x^{2}}=\frac{-2 b x}{\pi R^{2} \sqrt{R^{2}-x^{2}}}(x \neq \pm R)
$$

According to the derivation above, when $x \rightarrow R$, the twostep value of the out-of-focus QR code image is the largest. Through coordinate conversion, the position of the column where the maximum value of the two-step gradient, and the edge of the image when it is clear are located in the same coordinate system, the defocus radius can be determined by $R=x_{c}-x_{Q}$. In this equation, $x_{Q}$ is the position of the column where the maximum value of the two-step gradient.

Step 4: Restore the image. After obtaining the blur radius in the step 3, we introduce it into the defocusing degradation model to obtain the PSF. Then, the Wiener filtering algorithm
$[16,51]$ is used to restore, and the restoration results are binarized. Not only can this method enhance the anti-noise performance, but improve the restoration performance.

Combined with all the steps, the overall flow of the algorithm is shown in Fig. 9.

Through the analysis above, we can quickly restore the outof-focus blurred QR code images. Moreover, to verify the effectiveness of the algorithm, the specific experimental analysis and results are shown in Section IV.

\section{EXPERIMENTAL RESULtS AND ANALYSIS}

The experiments mainly use QR codes with error correction level $\mathrm{M}$, and the experimental environment is Intel i7 processor, CPU 3.20GHz, and Matlab2018a. The parameters of contrast algorithms are lambda_grad $=4 \mathrm{e}-3$, kernel_size $=31$, kappa $=2.0$, lambda_dark $=4 \mathrm{e}-3$, border $=30$, lambda_l0 $=2 \mathrm{e}-4$, $w t \_l 0=6 \mathrm{e}-3, w t$ deconv $=2 \mathrm{e} 3, k s=15$, and $w t_{-} k e r=0.1$. After obtaining the blur radius, the paper uses the Wiener filter algorithm $[16,51]$ to restore the image and sets the noise to signal ratio (NSPR) to 0.001. For synthetic images, after obtaining the restored image, we binarize the output image to enhance the edges and improve the recognition rate. We also deblurred the real images, which were taken by a Nikon N90 SLR camera. To verify the superiority of the restoration method proposed in this paper, data comparison and analysis are carried out from the three dimensions of restoration effect, recovery speed and recognition rate. 
(1)

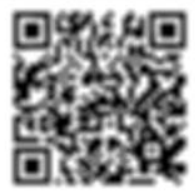

(2)

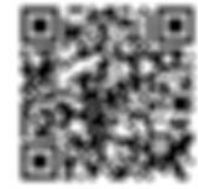

(3)

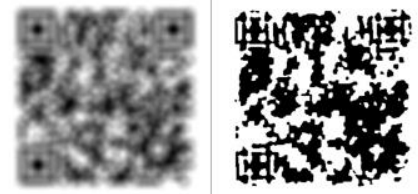

(4)

(5)

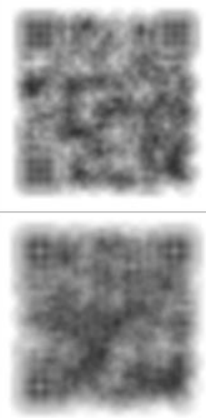

(a)
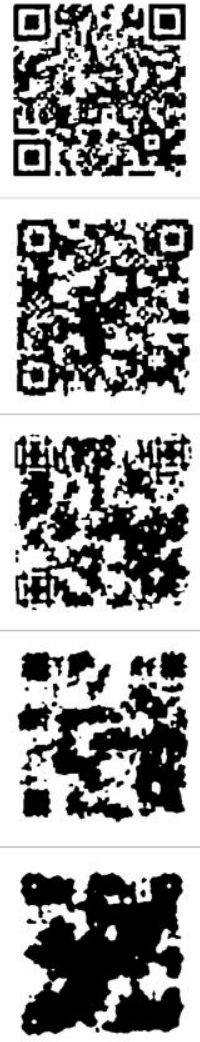

(b)
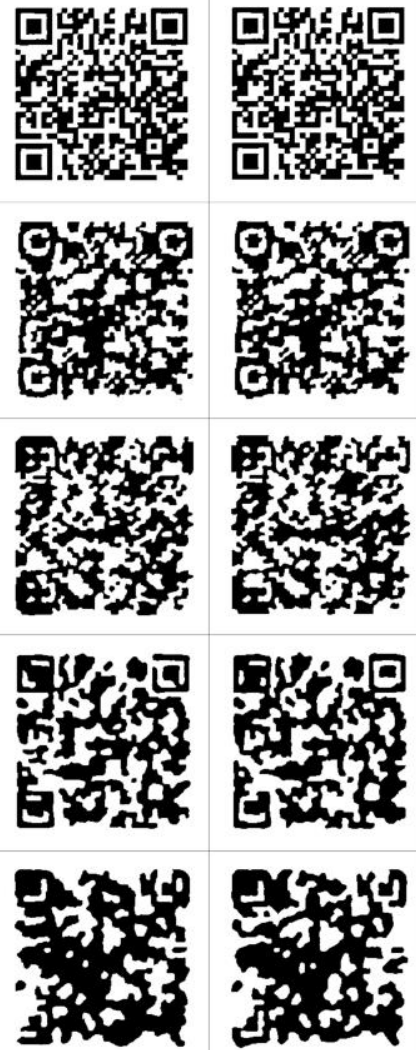

(c)
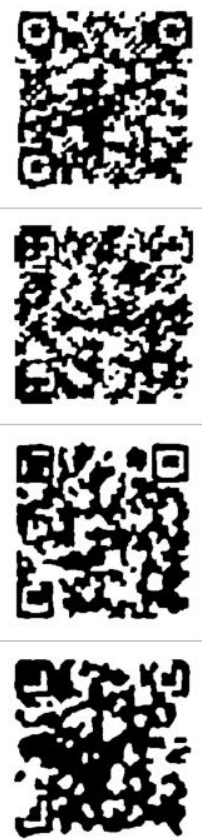

(d)
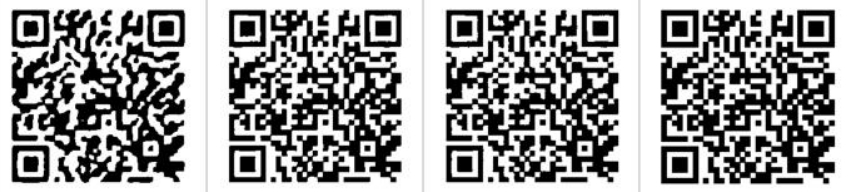
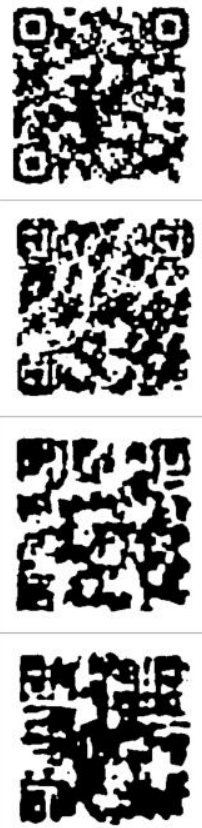

(e)
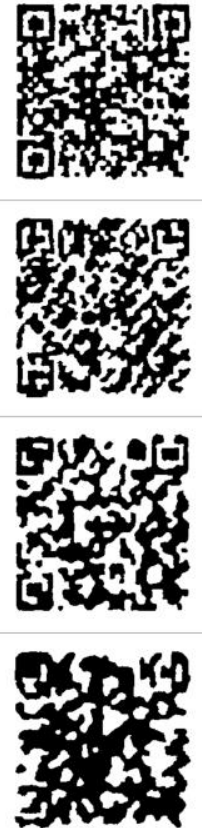

(f)
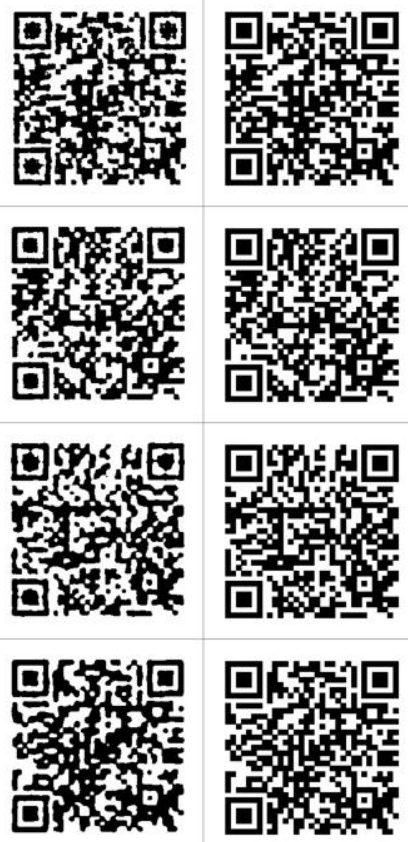

(g)

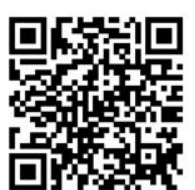

(h)

Fig. 10. Five deblurring samples of six different algorithms. (a) Synthetic blurred QR code (b) Xu et al. [23] (c) Pan et al. [6] (d) Yan et al. [44] (e) Bai et al. [27] (f) Wen et al. [45] (g) Ours (h) Ground truth.

\section{A. Deblurring Results on Synthetic QR Code Images}

In this section, the sizes of input images are normalized to $300 \times 300$ pixels, and the out-of-focus is added by MATLAB. The number of clear images in the common batch is $Q t y=12$. First of all, five QR codes with different blur degrees are selected and deblurred by different algorithms, as shown in Fig. 10. After that, the restoration effects of them by PSNR [52] and SSIM [53] are compared, as shown in Table $\|$. Combining with Fig. 10 and Table II, we can see that different algorithms have different restoration effects. In comparison, we can clearly know that our algorithm has certain advantages. For QR codes with a higher degree of blurred, the contrast of restoration effects is more obvious. When the blurred degree is small, Pan's algorithm [6], Yan's algorithm [44], and Wen's algorithm [45] have well recovery effects and recognizable. However, when the QR codes become more obscure, only our algorithm has a better recovery effect. It is because other restoration algorithms fail to consider the characteristics of QR codes fully, i.e., the edge characteristics, which results in the difficulty of solving the QR codes with a higher degree of blurred.

TABLE ॥

COMPARISON OF PSNR AND SSIM OF FIVE FIGURES

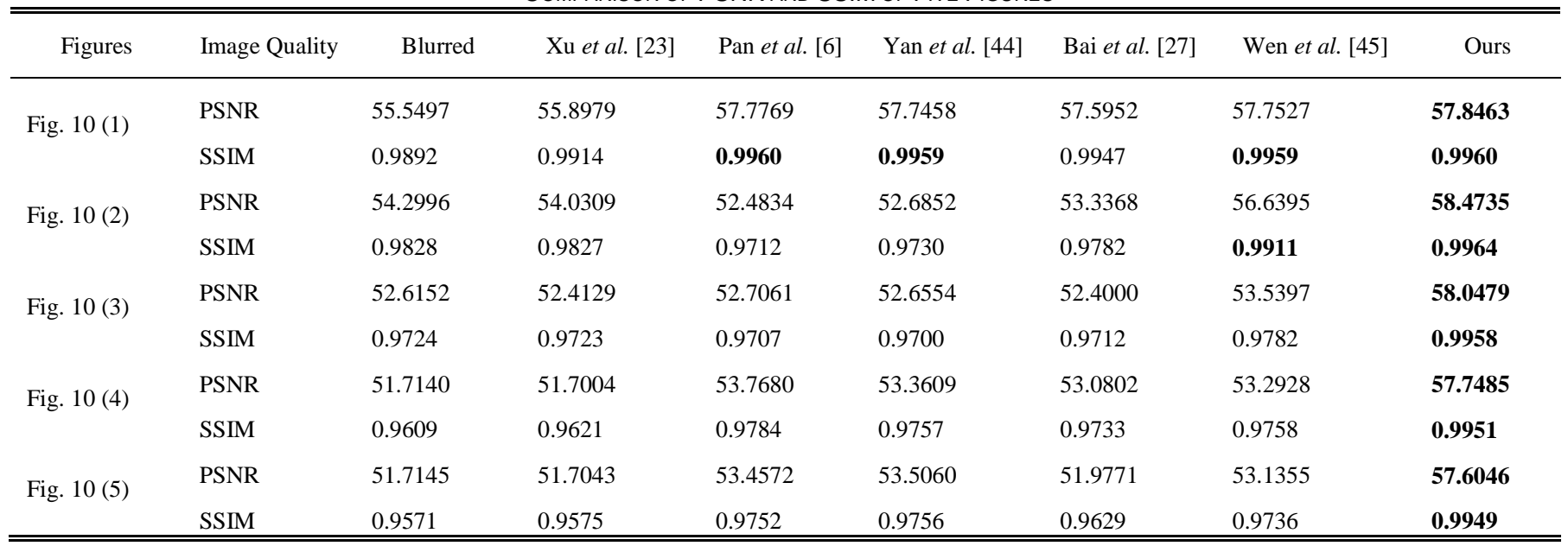




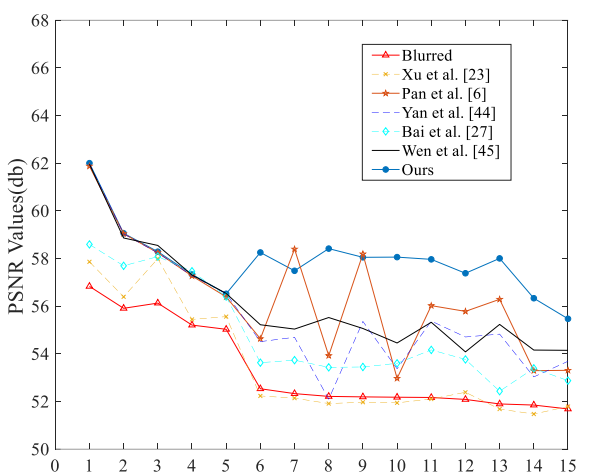

(a)

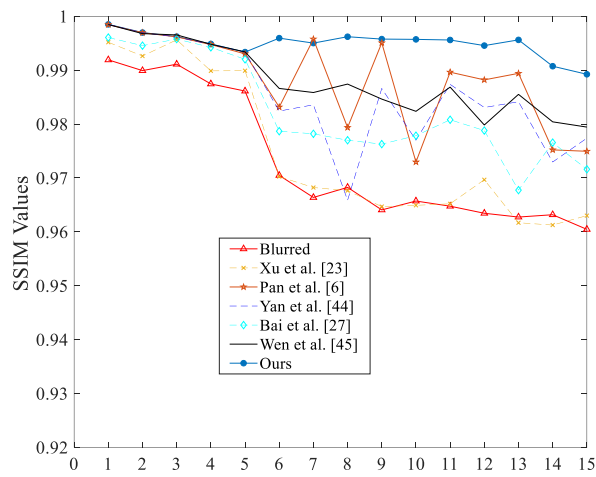

(b)

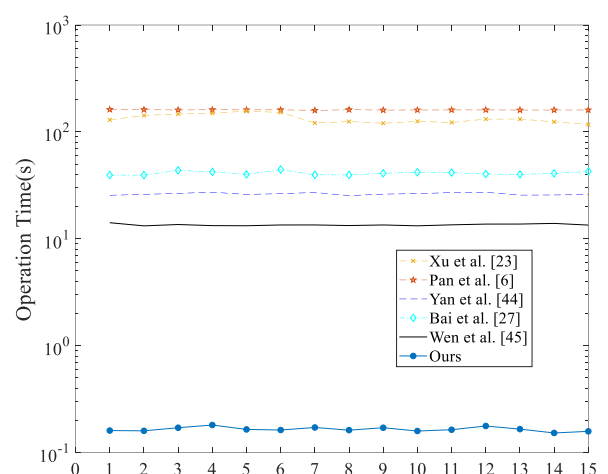

(c)

Fig. 11. Comparison of different restoration algorithms. (a)PSNR comparison, (b)SSIM comparison, (c)Operation time comparison.

\section{B. Recognition Rate and Speed}

Moreover, we move forward a step to start from other aspects, i.e., the recovery speed and recognition rate of different algorithms. The recognition rate indicates the proportion of the recovered images that can be recognized in the total test images, so does the recognition accuracy. In this part, we choose 15 QR codes with different blur degrees as well. The effects of restoration of different algorithms are compared in Fig. 11(a), Fig.11(b), and the operation time is shown in Fig. 11(c).

As shown in Fig. 11(a), Fig. 11(b), in terms of restoration effect, Pan's algorithm [6], Yan's algorithm [44], and Wen's algorithm [45] perform well. In Fig.11(c), Wen's algorithm [45] has a higher speed, which takes a shorter time to deblur. The reason for the slow speed of other algorithms is that there are many iterations, and the characteristics of QR code images
TABLE III

Comparison of Recognition Rate and Average Operation Time of Six Algorithms

\begin{tabular}{|c|c|c|c|c|}
\hline \multirow[b]{2}{*}{ Algorithms } & \multicolumn{3}{|c|}{ Recognition Rate } & \multirow[b]{2}{*}{$\begin{array}{c}\text { Average } \\
\text { Operation } \\
\text { Time/s }\end{array}$} \\
\hline & $\begin{array}{c}\text { Zxing } \\
\text { (v3.4.0) }\end{array}$ & $\begin{array}{l}\text { WeChat } \\
(\mathrm{v} 8.0 .2)\end{array}$ & $\begin{array}{c}\text { QR Code } \\
\text { Reader } \\
(\mathrm{v} 2.3 .25)\end{array}$ & \\
\hline None & $30.38 \%$ & $61.54 \%$ & $61.54 \%$ & --- \\
\hline Xu et al. [23] & $62.12 \%$ & $63.08 \%$ & $64.23 \%$ & 134.3274 \\
\hline Pan et al. [6] & $82.31 \%$ & $82.50 \%$ & $82.69 \%$ & 163.0592 \\
\hline Yan et al. [44] & $80.96 \%$ & $81.35 \%$ & $81.35 \%$ & 26.2886 \\
\hline Bai et al. [27] & $64.62 \%$ & $67.12 \%$ & $67.31 \%$ & 40.7498 \\
\hline Wen et al. [45] & $83.85 \%$ & $84.42 \%$ & $84.42 \%$ & 19.9678 \\
\hline Ours & $85.19 \%$ & $95 \%$ & $95.38 \%$ & 0.1760 \\
\hline
\end{tabular}

(1)

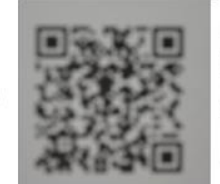

(2)

(3)
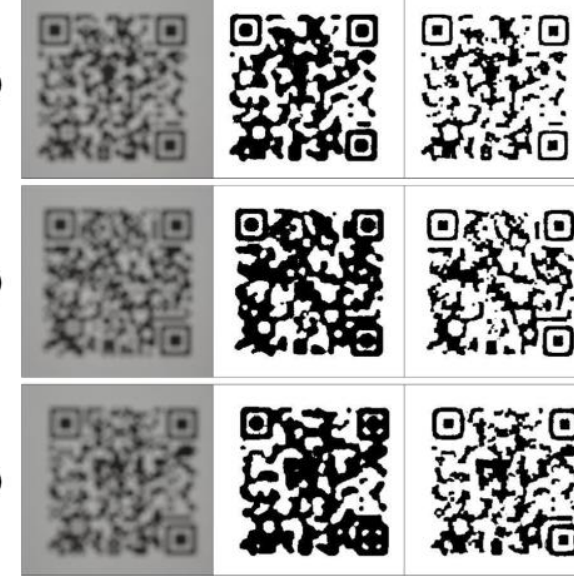

(4)

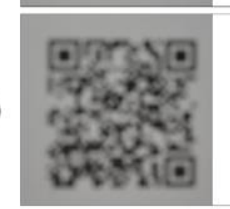

(5)

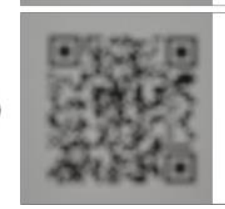

(a)
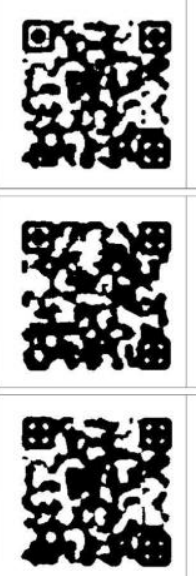

(b)
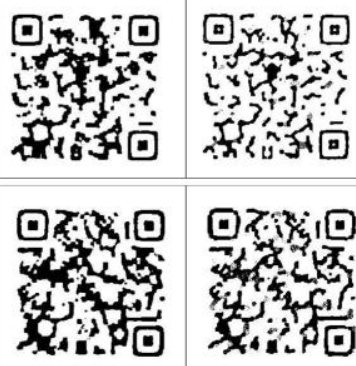

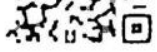
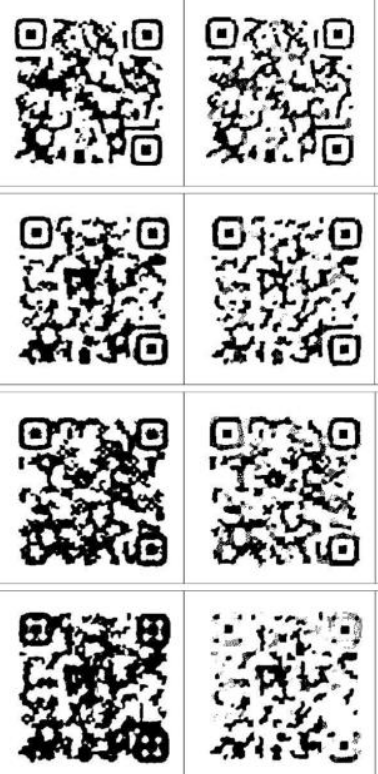

(c)


(d)
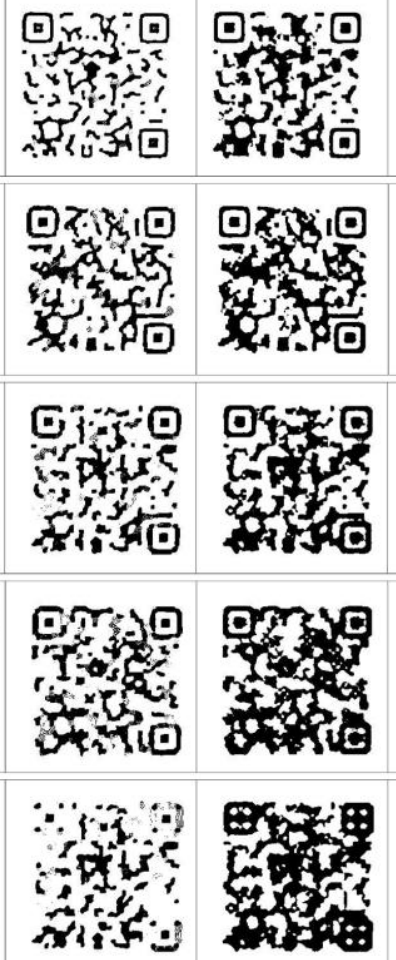

(e)

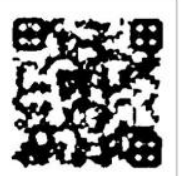

(f)
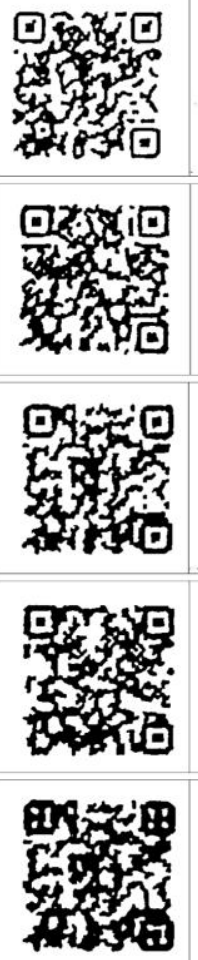

(g)
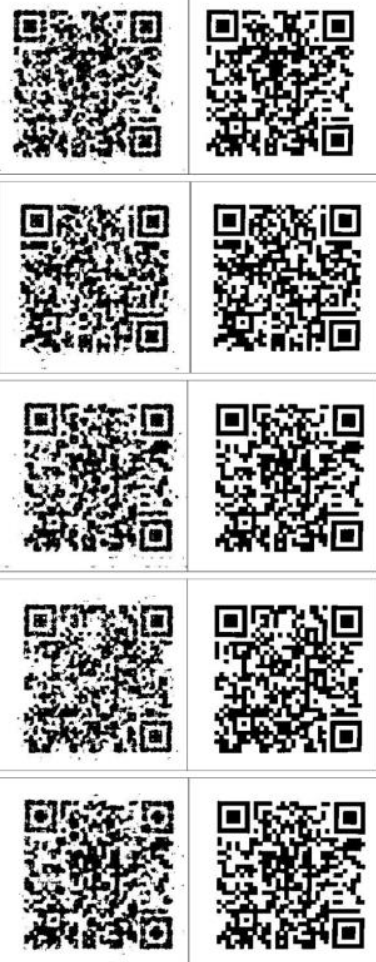

(h)

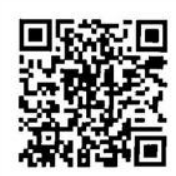

(i)

Fig. 12. Comparison of different restoration algorithms on real out-of-focus images. (a) Real blurred QR code (b) Binary image of (a) (c) Xu et al. [23] (d) Pan et al. [6] (e) Yan et al. [44] (f) Bai et al. [27] (g) Wen et al. [45] (h) Ours (i) Ground truth. 
(1)

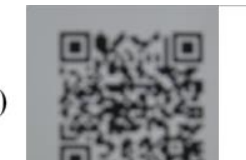

(2)



(3)



(4)

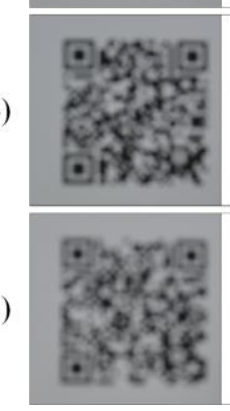

(a)
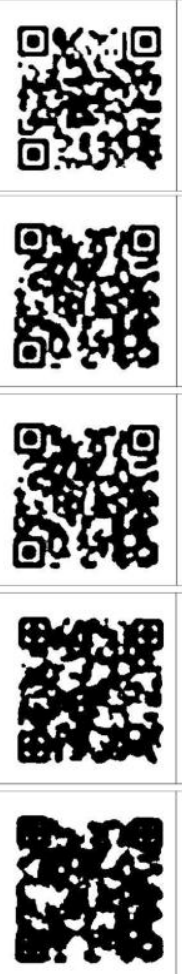

(b)
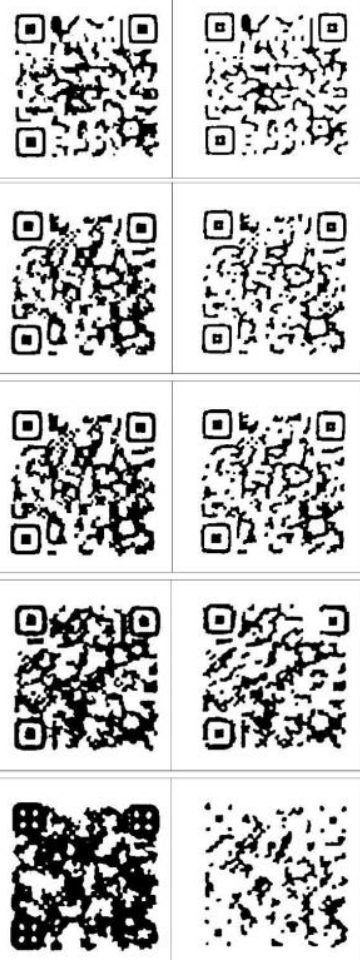

(c)
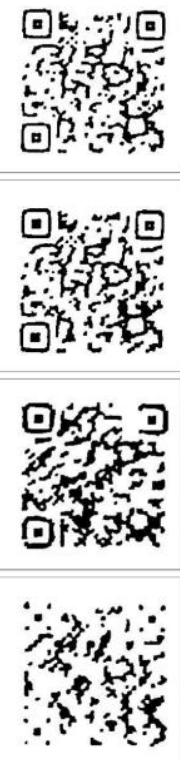

(d)
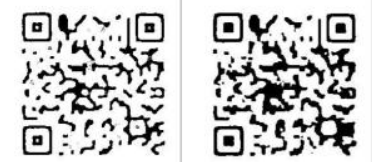
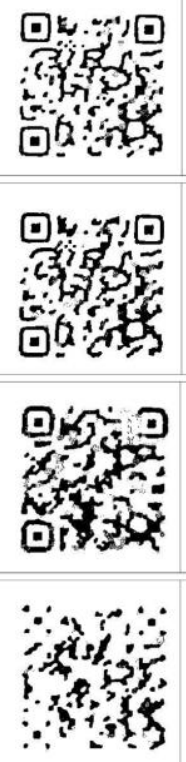

(e)
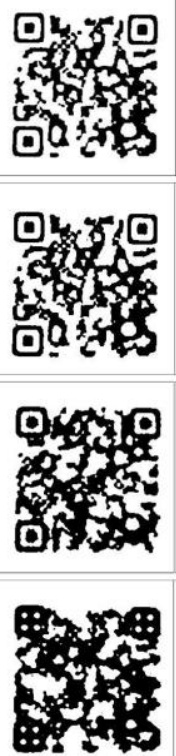

(f)
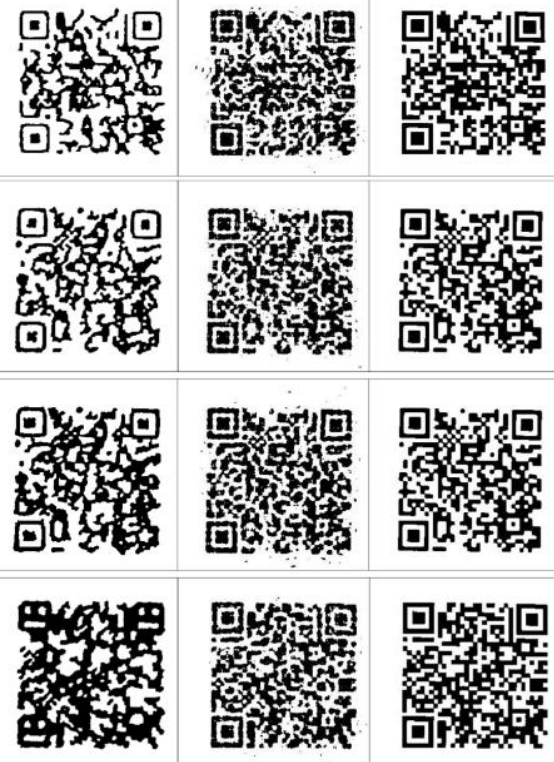

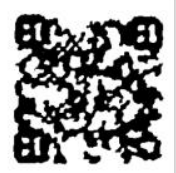

(g)

Fig. 13. Comparison of different restoration algorithms on another real out-of-focus images. (a) Real blurred QR code (b) Binary image of (a) (c) Xu et al. [23] (d) Pan et al. [6] (e) Yan et al. [44] (f) Bai et al. [27] (g) Wen et al. [45] (h) Ours (i) Ground truth.

TABLE IV

COMPARISON OF PSNR AND SSIM OF FIVE FIGURES

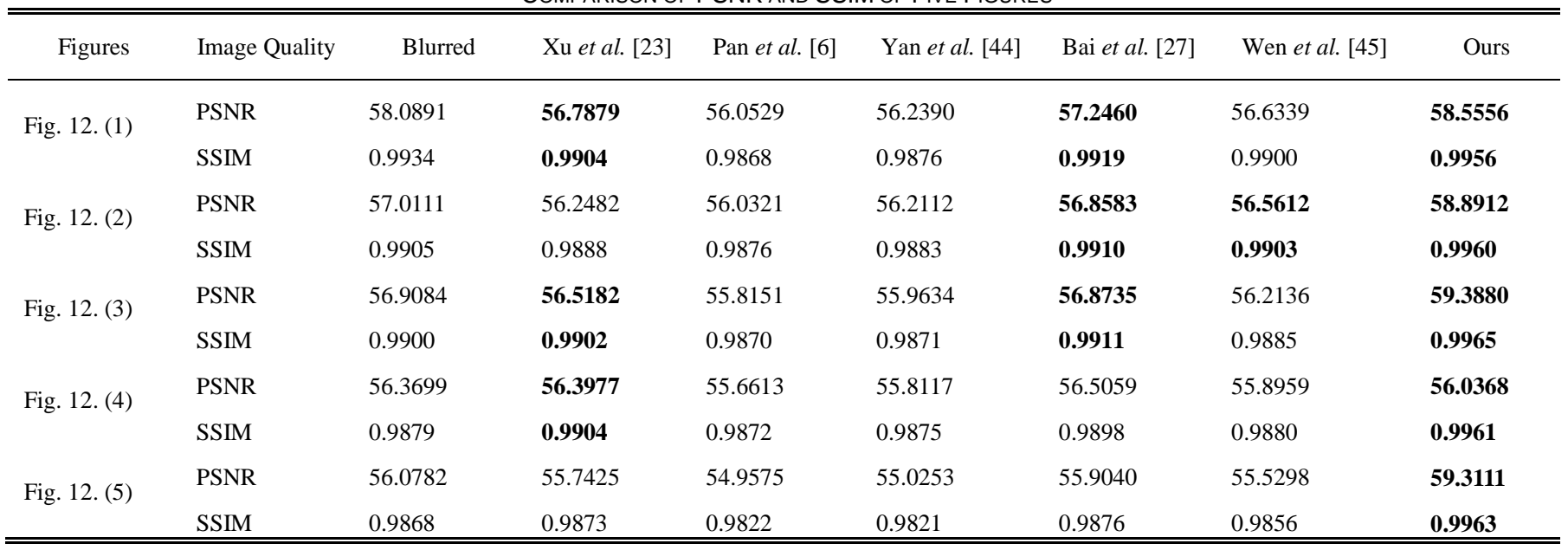

are not considered fully. However, our algorithm introduces the edge prior information of the image to convert the blind image restoration problem into a non-blind restoration problem, and then deblur through the Wiener filtering algorithm. In other words, as long as the blur radius can be accurately calculated, not only the restoration effect is guaranteed, but the recovery speed is greatly improved.

Last but not least, in order to verify the performance of the algorithms, this paper compares six different algorithms from the dimensions of average speed and recognition rate, and the results are shown in Table III. There are 520 experimental samples of QR codes under different blur degrees, including
320 images of lightly blurred, 120 images of moderately blurred, and 80 images of severely blurred.

It can be seen from Table III that in the comparison of different restoration algorithms, the recognition rate of Pan's algorithm [6] and Wen's algorithm [45] is relatively high, while the average operation time of Yan's algorithm [44] and Wen's algorithm [45] is relatively short. However, combining these two aspects, as for this sample set, the proposed algorithm, which only takes 0.176 seconds, is the fastest deblurring algorithm. These results illustrate that our algorithm can effectively restore blurred QR codes, especially for severe ones. 
TABLE $V$

COMPARISON OF PSNR AND SSIM OF FIVE FIGURES

\begin{tabular}{|c|c|c|c|c|c|c|c|c|}
\hline Figures & Image Quality & Blurred & $\mathrm{Xu}$ et al. [23] & Pan et al. [6] & Yan et al. [44] & Bai et al. [27] & Wen et al. [45] & Ours \\
\hline \multirow{2}{*}{ Fig. 13. (1) } & PSNR & 58.7547 & 56.7540 & 56.3030 & 56.4061 & $\mathbf{5 7 . 5 1 7 1}$ & 56.675 & $\mathbf{5 8 . 5 7 2 7}$ \\
\hline & SSIM & 0.9947 & 0.9896 & 0.9877 & 0.9881 & 0.9923 & 0.9904 & 0.9958 \\
\hline \multirow{2}{*}{ Fig. 13. (2) } & PSNR & 57.4648 & 56.9856 & 56.4556 & 56.8252 & $\mathbf{5 7 . 7 4 3 7}$ & 56.4591 & 58.4003 \\
\hline & SSIM & 0.9913 & 0.9912 & 0.9891 & 0.9903 & 0.9935 & 0.9902 & 0.9955 \\
\hline \multirow{2}{*}{ Fig. 13. (3) } & PSNR & 57.2383 & $\mathbf{5 7 . 3 1 1 2}$ & 56.7175 & 56.7735 & 57.7767 & 56.7914 & $\mathbf{5 7 . 6 7 7 1}$ \\
\hline & SSIM & 0.9905 & 0.9922 & 0.9902 & 0.9900 & 0.9933 & 0.9910 & 0.9939 \\
\hline \multirow{2}{*}{ Fig. 13. (4) } & PSNR & 56.3161 & 56.6515 & 55.6165 & 54.8326 & 56.7859 & 55.8864 & 59.6331 \\
\hline & SSIM & 0.9876 & 0.9904 & 0.9862 & 0.9812 & 0.9907 & 0.9874 & 0.9968 \\
\hline \multirow{2}{*}{ Fig. 13. (5) } & PSNR & 55.2280 & 55.1174 & 54.4871 & 54.6367 & 55.2782 & 54.9218 & 59.6226 \\
\hline & SSIM & 0.9817 & 0.9830 & 0.9784 & 0.9791 & 0.9840 & 0.9824 & 0.9933 \\
\hline
\end{tabular}

In summary, taking into account the above overall experimental results, for synthetic $\mathrm{QR}$ code images, the algorithm proposed in this paper has certain advantages in recognition rate and recovery speed. It lays an important foundation for real QR code images in the next section.

\section{Deblurring Results on Real QR Code Images}

Based on the experimental analysis of synthetic images, we further apply the proposed algorithm to actual application scenarios. We preprocess the real out-of-focus QR code images by unifying the image size to $300 \times 300$ pixels. In this part, we take two batches of QR codes as examples and conduct quantitative estimation. Fig. 12 shows the deblurring results on out-of-focus blurred QR code images of the common batch. In this batch, $Q t y=113$, and the prior value is prior $=112.625$. Fig. 13 shows the deblurring results of another batch of out-of-focus blurred $\mathrm{QR}$ code images. In this batch, $Q t y=89$, and the calculated prior value is prior $=111.1389$. Table IV and Table $V$ list the PSNR and SSIM values of different deblurring algorithms accordingly.

Ringing effects appear after deblurring, which will have an adverse impact on image quality or even recognizability. To address this difficulty, we use the Otsu algorithm [54] to binarize the restored image. For a fair comparison, we do the same for all algorithms. From the comparison of different algorithms in Fig. 12, Fig. 13, Table IV, and Table V, unlike the synthesized QR code images, Xu et al. [23], Bai et al. [27] and Wen et al. [45] have a better effect for the real images, which have more noise and are usually non-uniformly blurred. These traits are beneficial to each iteration of these algorithms. Bai et al. [27] has a better restoration effect by reconstructing the image in the frequency domain and the node domain. Pan et al. [6] is based on the fast convergence effect of the look-up table in practice and it does not have a very good competitive ability for real images. Yan et al. [44] is an improvement on Pan's algorithm [6], but the effect is not ideal as well. Wen's algorithm [45] is based on clear images with more patch-wise minimal pixels than blurred images and handles details better than other algorithms, but it is still not satisfactory for QR code images with a larger degree of blur. However, driven by the prior information of the common batch, it can be seen that the algorithm proposed in this paper can more effectively
TABLE VI

Comparison of Recognition Rate and Average Operation Time of Six Algorithms

\begin{tabular}{|c|c|c|c|c|}
\hline \multirow[b]{2}{*}{ Algorithms } & \multicolumn{3}{|c|}{ Recognition Rate } & \multirow[b]{2}{*}{$\begin{array}{c}\text { Average } \\
\text { Operation } \\
\text { Time/s }\end{array}$} \\
\hline & $\begin{array}{c}\text { Zxing } \\
\text { (v3.4.0) }\end{array}$ & $\begin{array}{l}\text { WeChat } \\
\text { (v8.0.2) }\end{array}$ & $\begin{array}{c}\text { QR Code } \\
\text { Reader } \\
(\mathrm{v} 2.3 .25)\end{array}$ & \\
\hline $\mathrm{Xu}$ et al. [23] & $62.76 \%$ & $85.29 \%$ & $79.88 \%$ & 126.5140 \\
\hline Pan et al. [6] & $61.26 \%$ & $77.18 \%$ & $65.17 \%$ & 146.8030 \\
\hline Yan et al. [44] & $61.26 \%$ & $78.68 \%$ & $69.67 \%$ & 23.3180 \\
\hline Bai et al. [27] & $66.37 \%$ & $84.95 \%$ & $81.68 \%$ & 21.3669 \\
\hline Wen et al. [45] & $62.76 \%$ & $85.59 \%$ & $79.98 \%$ & 13.3958 \\
\hline Ours & $63.06 \%$ & $91.59 \%$ & $88.59 \%$ & 0.1396 \\
\hline
\end{tabular}

solve the difficulty of out-of-focus QR codes, especially for the severe ones.

\section{Recognition Accuracy and Speed}

We also compare the computational time and recognition accuracy of the algorithms. In this section, we choose 333 real QR code images, include 202 images of lightly blurred, and the results are shown in Table VI. Compared with Table III, six algorithms have less consuming time for severely uniformly blurred QR code images can be produced under ideal synthetic conditions, but the real images taken with an SLR cannot be realized. As seen in Table VI, Xu's algorithm [23], and Bai's algorithm [27] have higher recognition rate, but Bai et al. [27] and Wen et al. [45] spend less time. Xu et al. [23], and Pan et al. [6] are relatively slow, requiring 126.5, and 146.8 seconds, respectively. Wen's algorithm [45], which pays attention to the connected domain, is the fastest among the five comparison algorithms. In spite of the QR codes with a slight degree of blur have a high image restoration quality, the recognition effect is not satisfactory. However, the proposed algorithm is the fastest of all algorithms and only takes 0.14 seconds. Thanks to the fact that both edge prior information and parameter estimation are computationally efficient and the proposed algorithm does not require redundant iterative processes.

According to the visual effect of the restored images, recognition accuracy and the average operation time of both synthesized pictures and real images, our algorithm has great shown stability and robustness. In a word, the proposed algorithm is suitable for tackling out-of-focus blurred QR code 
images caused by optical imaging problems, especially for the barcode application systems.

\section{CONCLUSION}

Throughout this article, our focus has been on developing and testing a fast restoration method based on edge prior information for out-of-focus blurred images of $\mathrm{QR}$ code via image sensing. Combining with the edge characteristics, the blurred QR code image can be quickly restored under the guidance of CMOS sensing technology and optical information theory. The strength of our method is that it can efficiently estimate the parameters of PSF, thereby restoring the common batch of blurred images. Our results were tested on synthetic images and real images with light, moderate, severe amounts of blurring. It has been validated by experiments that have fully illustrated the efficiency and robustness of the proposed method. Intrinsically, a natural question is to what extend our method can be used for industrial applications, i.e., warehouse auto-sorting system. The operation time is short enough, which has great application potential in barcode application systems.

Nonetheless, the proposed algorithm has some limitations. The restoration effect would be affected while the QR code images with uneven blur, uneven illumination, or excessive distortion. Complex background is an important factor as well. In future work, we will focus on improving the applicability by combining the most state-of-art -techniques. E.g., spectraldomain feature segmentation [55] can extract the QR code images in a complex environment, and curvelet transform [56] is able to remove the undesirable artifacts introduced during the data acquisition process.

\section{REFERENCES}

[1] G. J. Chou, and R. -Z. Wang, "The Nested QR Code," IEEE Signal Process. Lett., vol. 27, pp. 1230-1234, Jul. 2020.

[2] A. Banu, K. Ganagavalli, and G. Ramsundar, "QR Code Based Shopping with Secure Checkout for Smartphones," J. Comput. Theor. Nanosci., vol. 15, no. 5, pp. 1545-1550, May. 2018.

[3] T. Yuan, Y. Wang, et al, "Two-layer QR codes," IEEE Trans. Image Process., vol. 28, no. 9, pp. 4413-4428, Sept. 2019.

[4] N. Liu, Y. Du, and Y. Xu, "QR codes blind deconvolution algorithm based on binary characteristic and L0 norm minimization," Pattern Recognit. Lett., vol. 111, pp. 117-123, Aug. 2018.

[5] H. Pu, M. Fan, J. Yang, and J. Lian, "Quick response barcode deblurring via doubly convolutional neural network," Multimed. Tools Appl., vol. 78, no. 1, pp. 897-912, Mar. 2018.

[6] J. Pan, D. Sun, H. Pfister, and M. -H. Yang, "Blind image deblurring using dark channel prior," in CVPR, Las Vegas, Nevada, 2016, pp.1628-1636.

[7] Y. Q. Liu, X. Du, H. L. Shen, and S. J Chen, "Estimating Generalized Gaussian Blur Kernels for Out-of-Focus Image Deblurring," IEEE Trans. Circuits Syst. Video Technol., vol. 31, no.3, Mar. 2021.

[8] A. Levin, R. Fergus, F. Durand, and W. T. Freeman, "Image and depth from a conventional camera with a coded aperture," ACM Trans. Graph., vol. 26, no. 3, pp. 70-es, Jul. 2007.

[9] Y. Van. Gennip, P. Athavale, J. Gilles, and R. Choksi, "A regularization approach to blind deblurring and denoising of QR barcodes," IEEE Trans. Image Process., vol. 24, no. 9, pp. 2864-2873, Sept. 2015.

[10] N. Liu, X. Zheng, H. Sun, and X. Tan, "Two-dimensional bar code outof-focus deblurring via the increment constrained least squares filter," Pattern Recognit. Lett., vol. 34, no. 2, pp. 124-130, Jan. 2013.

[11] S. Tiwari, "Blind restoration of motion blurred barcode images using ridgelet transform and radial basis function neural network," electronic letters on computer vision and image analysis (ELCVIA), vol. 13, no. 3 , pp. 63-80, Oct. 2014.
[12] T. Michaeli and M. Irani, "Blind deblurring using internal patch recurrence," in ECCV, Zurich, Switzerland, 2014, pp. 783-798.

[13] S. Nah, T. Hyun. Kim, and K. Mu. Lee, "Deep multi-scale convolutional neural network for dynamic scene deblurring," in CVPR, Honolulu, Hawaii, 2017, pp. 3883-3891.

[14] R. Chen, Y. Yu, X. Xu, L. Wang, H. Zhao, and H. -Z. Tan, "Adaptive Binarization of QR Code Images for Fast Automatic Sorting in Warehouse Systems," Sensors, vol. 19, no. 24, pp. 5466, Dec. 2019.

[15] Y. Bai, H. Jia, M. Jiang, et al, "Single-Image Blind Deblurring Using Multi-Scale Latent Structure Prior," IEEE Trans. Circuits Syst. Video Technol., vol. 30, no. 7, pp. 2033-2045, Jul. 2020.

[16] N. Wiener, "Extrapolation, interpolation, and smoothing of stationary time series with engineering applications," John Wiley \& Sons, 1950.

[17] W. H. Richardson, "Bayesian-based iterative method of image restoration," J. Opt. Soc. Am., vol. 62, no. 1, pp. 55-59, Jan. 1972.

[18] L. B. Lucy, "An iterative technique for the rectification of observed distributions," ASTRON J., vol. 79, no. 6, pp. 745-754, Jun. 1974.

[19] C. K. Liew, "Inequality constrained least-squares estimation," J. Am. Stat. Assoc., vol. 71, no. 355, pp. 746-751, 1976.

[20] T. Eboli, J. Sun, and J. Ponce, "End-to-end Interpretable Learning of Non-blind Image Deblurring," arXiv preprint arXiv:2007.01769, 2020.

[21] T. Adam, and R. Paramesran, "Hybrid non-convex second-order total variation with applications to non-blind image deblurring," Signal Image Video Process., vol. 14, no. 1, pp. 115-123, Jul. 2019.

[22] J. Zhang, J. Pan, W. S. Lai, R. W. Lau, and M. H. Yang, "Learning fully convolutional networks for iterative non-blind deconvolution," In CVPR, Honolulu, Hawaii, 2017, pp. 3817-3825.

[23] L. Xu, S. Zheng, and J. Jia, "Unnatural L0 sparse representation for natural image deblurring," in CVPR, Portland, Oregon, USA, 2013, pp. 1107-1114.

[24] J. Pan, Z. Hu, Z. Su, and M. H. Yang, "L0-regularized intensity and gradient prior for deblurring text images and beyond," IEEE Trans. Pattern Anal. Mach. Intell., vol. 39, no. 2, pp. 342-355, Feb. 2017.

[25] W. S. Lai, J. J. Ding, Y. Y. Lin, and Y. Y. Chuang, "Blur kernel estimation using normalized color-line priors," in CVPR, Boston, Massachusetts, USA, 2015, pp. 64-72.

[26] W. Ren, et al., "Image deblurring via enhanced low-rank prior," IEEE Trans. Image Process., vol. 25, no. 7, pp. 3426-3437, Jul. 2016.

[27] Y. Bai, G. Cheung, X. Liu, and W. Gao, "Graph-based blind image deblurring from a single photograph," IEEE Trans. Image Process., vol. 28, no. 3, pp. 1404-1418, Mar. 2019.

[28] L. Chen, F. Fang, T. Wang, and G. Zhang, "Blind image deblurring with local maximum gradient prior," in CVPR, Long Beach, CA, 2019, pp. $1742-1750$.

[29] D. Ren, K. Zhang, et al, "Neural blind deconvolution using deep priors," in CVPR, Seattle, WA, US, 2020, pp. 3341-3350.

[30] Y. Nan, and H. Ji, "Deep Learning for Handling Kernel/model Uncertainty in Image Deconvolution," in CVPR, Seattle, WA, US, 2020, pp. 2388-2397.

[31] D. Ren, W. Zuo, D. Zhang, L. Zhang, and M. H. Yang, "Simultaneous fidelity and regularization learning for image restoration," IEEE Trans. Pattern Anal. Mach. Intell., vol. 43, no. 1, pp. 284-299, Jan. 2021.

[32] J. Zhang, J. Pan, J. Ren, Y. Song, L. Bao, R. W. Lau, and M.-H. Yang, "Dynamic scene deblurring using spatially variant recurrent neural networks," in CVPR, Salt Lake City, Utah, 2018, pp. 2521-2529.

[33] O. Kupyn, V. Budzan, M. Mykhailych, D. Mishkin, and J. Matas, "Deblurgan: Blind motion deblurring using conditional adversarial networks," in CVPR, Salt Lake City, Utah, 2018, pp. 8183-8192.

[34] X. Tao, H. Gao, et al, "Scale-recurrent network for deep image deblurring," in CVPR, Salt Lake City, Utah, 2018, pp. 8174-8182.

[35] G. Rioux, C. Scarvelis, R. Choksi, T. Hoheisel, and P. Marechal, "Blind deblurring of barcodes via Kullback-Leibler divergence," IEEE Trans. Pattern Anal. Mach. Intell., vol. 43, no. 1, pp. 77-88, Jan. 2021.

[36] R. Choksi, Y. van Gennip, and A. Oberman, "Anisotropic total variation regularized $L^{1}$-approximation and denoising/deblurring of $2 \mathrm{D}$ bar codes," Inverse Problems Imag., vol. 5, no. 3, pp. 591-617, Mar. 2011.

[37] A. El Gamal, and H. Eltoukhy, "CMOS image sensors," IEEE Circuits Syst. Mag., vol. 21, no, 3, pp. 6-20, Jun. 2005.

[38] W. Guan, Y. Wu, C. Xie, L. Fang, X. Liu, and Y. Chen, "Performance analysis and enhancement for visible light communication using CMOS sensors," Opt. Commun., vol. 410, pp. 531-551, Mar. 2018.

[39] M. Lebl, F. Sroubek, and J. Flusser, "Blur-Invariant Similarity Measurement of Images," IEEE Trans. Pattern Anal. Mach. Intell., early access, Nov. 2020. 
[40] J. Flusser, T. Suk, and Saic, S, "Recognition of blurred images by the method of moments," IEEE Trans. Image Process., vol. 5, no. 3, pp. 533-538, Mar. 1996.

[41] J. Flusser, and T. Suk, "Degraded image analysis: an invariant approach," IEEE Trans. Pattern Anal. Mach. Intell., vol. 20, no. 6, pp. 590-603, Jun. 1998.

[42] R. Chen, et al, "A Fast Lens Focus Measurement Method Based on Blurred Image Processing," Acta Opt. Sin., vol. 35, no. 2, pp. 107-115, Feb. 2015

[43] H. G. Jung, et al, "Coaxial optical structure for iris recognition from a distance,” Opt. Eng., vol. 50, no. 5, pp. 053201, May. 2011.

[44] Y. Yan, W. Ren, Y. Guo, and R. Wang, "Image deblurring via extreme channels prior," in CVPR, Honolulu, Hawaii, 2017, pp. 4003-4011.

[45] F. Wen, R. Ying, Y. Liu, P. Liu, and T. -K. Truong, "A simple local minimal intensity prior and an improved algorithm for blind image deblurring," IEEE Trans. Circuits Syst. Video Technol., early access, Oct. 2020 .

[46] K. Zhang, W. Zuo, and L. Zhang, "FFDNet: Toward a fast and flexible solution for CNN-based image denoising," IEEE Trans. Image Process., vol. 27, no. 9, pp. 4608-4622, Sept. 2018.

[47] A. Seif, M. M. Salut, and M. N. Marsono, "A hardware architecture of Prewitt edge detection," in 2010 IEEE Conference on Sustainable Utilization and Development in Engineering and Technology (STUDENT), Kuala Lumpur, Malaysia, 2010, pp. 99-101.

[48] R. Muthukrishnan and M. Radha, "Edge detection techniques for image segmentation," Int. J. Comput. Sci. Inform. Technol., vol. 3, no. 6, pp. 259-267, Dec. 2011.

[49] W. Gao, X. Zhang, L. Yang, and H. Liu, "An improved Sobel edge detection," in 2010 3rd International conference on computer science and information technology (ICCSIT), Chengdu, 2010, pp. 67-71.

[50] P. Bao, L. Zhang, and X. Wu, "Canny edge detection enhancement by scale multiplication," IEEE Trans. Pattern Anal. Mach. Intell., vol. 27, no. 9, pp. 1485-1490, Sept. 2005.

[51] K. Panetta, L. Bao, and S. Agaian, "Sequence-to-sequence similaritybased filter for image denoising," IEEE Sens. J., vol. 16, no. 11, pp. 4380-4388, Jun. 2016.

[52] W. Ye, S. Li, et al, "AK times singular value decomposition based image denoising algorithm for DoFP polarization image sensors with Gaussian noise," IEEE Sens. J., vol. 18, no. 15, pp. 6138-6144, Aug. 2018.

[53] H. Choi, and J. Jeong, "Despeckling images using a preprocessing filter and discrete wavelet transform-based noise reduction techniques," IEEE Sens. J., vol. 18, no. 8, pp. 3131-3139, Apr. 2018.

[54] N. Otsu, "A threshold selection method from gray-level histograms," IEEE T. Syst. Man. Cyb., vol. 9, no. 1, pp. 62-66. Jan. 1979.

[55] J. Zabalza, et al, "Novel two-dimensional singular spectrum analysis for effective feature extraction and data classification in hyperspectral imaging," IEEE Trans. Geosci. Remote Sens., vol. 53, no. 8, pp. 44184433, Feb. 2015.

[56] T. Qiao, et al, "Effective denoising and classification of hyperspectral images using curvelet transform and singular spectrum analysis," IEEE Trans. Geosci. Remote Sens., vol. 55, no. 1, pp 119-133, Nov. 2016.

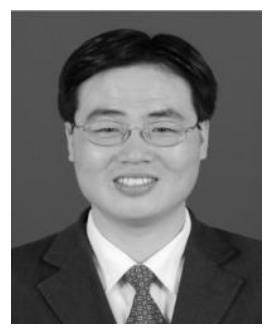

Rongjun Chen received his M.S. degree in Control Theory and Control Engineering from Guangdong University of Technology, China in 2007 and his Ph.D. degree in Communication and Information System from Sun Yat-sen University, China in 2015. He is currently an associate professor in the School of Computer Science, Guangdong Polytechnic Normal University, China. His research interests include image perception and processing and Internet

of things.

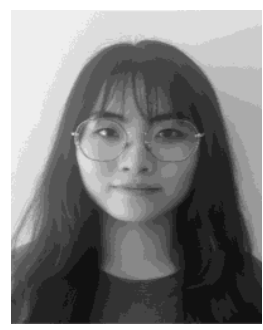

Zhijun Zheng is currently studying in control science and engineering at Guangdong Polytechnic Normal University, Guangzhou, China for her M.Eng degree. Her research interests mainly focus on image processing and perception and Internet of Things technology.

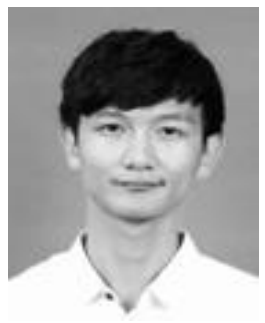

Yongxing $\mathrm{Yu}$ is studying for his master's degree in control science and engineering at Guangdong Polytechnic Normal University, Guangzhou, China. He is currently in the third year of graduates study. His research interests are Internet of Things technology, as well as image perception and processing.



Huimin Zhao received the B.Sc. and M.Sc. degrees in signal processing from Northwestern Polytechnical University, Xian, China, in 1992 and 1997, respectively, and the Ph.D. degree in electrical engineering from Sun Yat-sen University, in 2001. He is currently a Professor with Guangdong Polytechnic Normal University. His research interest includes image, video, and information security technology.

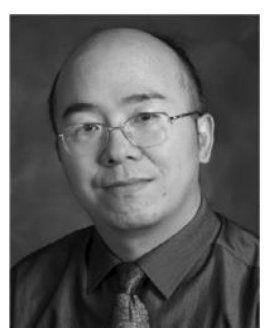

Jinchang Ren (Senior Member, IEEE) received the B.E. in computer software, M.Eng. in image processing, and the D.Eng. in computer vision from Northwestern Polytechnical University, Xian, China. He also received a Ph.D. degree in electronic imaging and media communication from the University of Bradford, Bradford, U.K. $\mathrm{He}$ is a Professor with the National Subsea Centre, Robert Gordon University, U.K., and he is also a Visiting Professor with GPNU. He has published about 300 articles. His research interests include computer vision and multimedia signal processing, especially on hyperspectral imaging, machine learning and big data analytics. He acts as Associate Editor for five international journals including IEEE Trans. Geoscience and Remote Sensing, Journal of the Franklin Institute, IET Image Processing and Big Data Analytics etc.

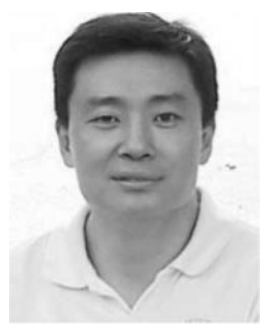

Hong-Zhou Tan (Senior Member, IEEE) received the Ph.D. degree in electronic engineering from the City University of Hong Kong, Hong Kong, and South China University of Technology, Guangzhou, China, in 1998. He was with several universities and IT companies in Hong Kong, Singapore, and Canada, from 1998 to 2004. Since 2004, he has been a full professor with the School of Electronics and Information Technology, Sun Yat-sen University, Guangzhou, China. His current research interests include the Internet of Things and energy harvesting. 